\title{
Efecto de la composición racial sobre la calidad espermática de verracos ${ }^{1}$
}

\section{Effect of breed composition on sperm quality of boar}

\author{
Anthony Valverde ${ }^{2}$, Mónica Madrigal-Valverde ${ }^{2,3}$, Marlen Camacho-Calvo ${ }^{2}$,Adones Zambrana-Jiménez ${ }^{2}$, \\ Leonardo López ${ }^{2}$
}

\begin{abstract}
Resumen
La evaluación de la calidad seminal constituye una herramienta para mejorar los índices reproductivos en las granjas porcinas. La movilidad del espermatozoide es un parámetro que se utiliza en la evaluación de la calidad espermática, porque se relaciona con el nivel de energía del gameto masculino. El objetivo de este estudio fue determinar la influencia de la composición racial sobre variables reproductivas de calidad, cinética y movilidad del semen en verracos. Durante el año 2016, se recolectaron 240 eyaculados de 63 verracos con una edad media de $24,4 \pm 10,9$ meses. Se identificaron seis grupos raciales: Duroc (D), Yorkshire (Y), Landrace (L), F1 Pietrain*Duroc (PD) y dos líneas genéticas (LA y LB). Los grupos raciales con mayor volumen de eyaculado presentaron un número total de espermatozoides más elevado $(\mathrm{P}<0,05)$. El efecto del grupo racial fue significativo $(\mathrm{P}<0,05)$ sobre las variables de cinética espermática, excepto para la amplitud del desplazamiento lateral de la cabeza (ALH, $\mu \mathrm{m})$. La raza Landrace presentó el mayor $(\mathrm{P}<0,05)$ porcentaje de espermatozoides estáticos $(29,30 \pm 1,57)$. Las diferencias más relevantes $(\mathrm{P}<0,05)$ para motilidad total (MTOT) y mótiles progresivos (MP), se presentaron entre L y PD con valores de $70,71 \pm 1,57 ; 77,48 \pm 1,09$ y $51,80 \pm 1,97 ; 59,85 \pm 1,37 \%$, respectivamente. Los verracos adultos $(\geq 18$ meses) presentaron mayores volúmenes de eyaculado y número total de espermatozoides con respecto a los verracos de edades intermedias y jóvenes, sin embargo, para las velocidades ( $\mu \mathrm{m} / \mathrm{s})$ : curvilínea (VCL), rectilínea (VSL) y promedio (VAP), los verracos adultos fueron distintos $(\mathrm{P}<0,05)$ de los verracos en edad intermedia. Se identificaron cuatro subpoblaciones espermáticas (SP), SP1 $(46,83 \%)$ caracterizadas por una velocidad moderada, pero de movimiento muy progresivo, SP2 (14,78\%) con movimiento activo, pero no progresivo, SP3 $(8,45 \%)$ con velocidades bajas y trayectorias progresivas y SP4 $(29,94 \%)$ con movimiento muy rápido, pero sin progresividad.
\end{abstract}

Palabras clave: cerdo, espermatozoo, semen, reproducción.

\begin{abstract}
The assessment of the semen quality is an essential tool to improve the reproductive indexes in swine farms. The motility is the most important parameter that used in the sperm quality evaluation because it is associate to the energy of the spermatozoon. The aim of this research was to determine the influence of breed composition on reproductive

1 Recibido: 19 de febrero, 2018. Aceptado: 30 de mayo, 2018. Este trabajo formó parte del proyecto de investigación 2151-056, inscrito en la Vicerrectoría de Investigación y Extensión del Instituto Tecnológico de Costa Rica (ITCR), San Carlos, Costa Rica.

2 Instituto Tecnológico de Costa Rica (ITCR), Escuela de Agronomía, Centro de Investigación y Desarrollo en Agricultura Sostenible del Trópico Húmedo, Sede Regional San Carlos. Apdo. Postal 223-21001 Alajuela, Costa Rica. anvalverde@itcr.ac.cr (autor para correspondencia); mcamacho@itcr.ac.cr; azambranaj1@yahoo.es; leonardolopezsanchez4@gmail.com

3 Becaria de la Organización de Estados Americanos-Grupo Coimbra de Universidades Brasileñas (OEA-GCUB-CAPES). Grupo de Estudos de Reprodução Animal, Universidade Federal da Bahia. Apdo. Postal 40170-11, Bahía, Brasil. madrigalvalverdem@gmail.com
\end{abstract}


variables of sperm quality, kinetics and semen motility in boars. During 2016, 240 ejaculates were collected from 63 boars with a mean age of $24.4 \pm 10.9$ months. Six racial groups were identified: Duroc (D), Yorkshire (Y), Landrace (L), F1 Pietrain*Duroc (PD) and two genetic lines (LA and LB). The breeds with the highest ejaculate volume presented a higher total number of spermatozoa $(\mathrm{P}<0.05)$. The effect of the breed was significant $(\mathrm{P}<0.05)$ on sperm kinetics variables except for amplitude of lateral head displacement (ALH, $\mu \mathrm{m})$. The Landrace breed presented the highest $(\mathrm{P}<0.05)$ percentage of static sperm $(29.30 \pm 1.57)$. The most relevant differences $(\mathrm{P}<0.05)$ for total motility (MTOT) and progressive motility (MP), were presented between L and PD with values of 70.71 $1.57 ; 77.48 \pm 1.09$ and $51.80 \pm 1.97 ; 59.85 \pm 1.37 \%$ respectively. Adult boars ( $\geq 18$ months) had higher volumes of ejaculate and total number of sperm than boars in the intermediate and young ages, however, for velocities $(\mu \mathrm{m} / \mathrm{s})$ : curvilinear (VCL), straight line (VSL) and average path (VAP), adult boars only were different $(\mathrm{P}<0.05)$ of the boars in-between ages. Four sperm subpopulations (SP) were identified, SP1 (46.83\%) characterized by a moderate speed, but of very progressive motility, SP2 (14.78\%) with active movement, but not progressive, SP3 (8.45\%) with low speeds and progressive path and SP4 (29.94\%) with quick movement, but without progressivity.

Keywords: swine, spermatozoa, semen, reproduction.

\section{Introducción}

La industria porcina se ha caracterizado por implementar la inseminación artificial en granjas comerciales, con verracos seleccionados con base en caracteres de tipo cuantitativo y cualitativo, lo que resulta en un rápido progreso genético (Dyck et al., 2011). Sin embargo, esta selección se realiza casi exclusivamente para características de crecimiento y calidad de canal, con una importancia ponderada muy baja a caracteres como la calidad del semen (Robinson y Buhr, 2005). Esto implica que no es conocido el mérito genético de los verracos a nivel comercial, en relación con sus características reproductivas. La calidad seminal constituye una forma de seleccionar e identificar los verracos (Okere et al., 2005). El conocimiento de la fisiología, la espermatogénesis, la cinética espermática, la influencia de la raza y las características individuales de los verracos, podrían contribuir al éxito de la eficiencia reproductiva y la heredabilidad de rasgos genotípicos asociados a características deseables del semen porcino. Desde el punto de vista de comercialización de dosis seminales, la cantidad y la calidad del semen son los dos factores principales que afectan el número de dosis que se pueden producir por eyaculado (Flowers, 1997).

El desarrollo de la industria porcina por inseminación artificial (IA), ha llevado a mejoras en el análisis cuantitativo de muestras de semen de verraco para predecir el potencial de fertilidad de las dosis seminales. El análisis subjetivo basado en la experiencia técnica, ha mostrado un alto grado de variación en los resultados finales. Para evitar este problema, a partir de los años 80 del siglo pasado, se desarrolló una tecnología de Análisis de Semen Asistido por Computadora (CASA) para mejorar la exactitud y precisión del análisis de semen (Tardif et al., 1999; Gil et al., 2009; Bompart et al., 2018). Incluso el número de sistemas CASA aumenta continuamente en los centros de inseminación artificial (IA), donde es necesario estandarizar los métodos para la evaluación objetiva de la calidad del esperma (Verstegen et al., 2002; Amann y Katz, 2004; Gil et al., 2009).

Las características seminales como volumen, concentración y movilidad presentan variabilidad entre y dentro de razas. La variabilidad espermática, que es función de factores exógenos y endógenos (Ciereszko et al., 2000), debe ser caracterizada para evaluar la calidad del semen (Smital, 2009). La calidad espermática se estima por medio del análisis de semen y permite predecir la fertilidad potencial de un verraco reproductor. Un análisis general se basa en la estimación de la concentración espermática, la morfología y la calidad del movimiento con parámetros cinéticos. Además, el estudio de las causas de la baja fertilidad en las granjas se puede asociar con los análisis 
seminales. Sin embargo, algunos autores han reportado que las características seminales no están correlacionadas con la fertilidad de los machos (Tsakmakidis et al., 2010) y otros indican que la evaluación de la calidad del semen está asociada con la preñez (López-Rodríguez et al., 2013). Aún se desconocen las causas de la infertilidad en verracos, debido a que, cuando se identifica un macho con problemas de tipo reproductivo, la solución que se toma es sacrificarlo sin antes mediar algún examen y/o tratamiento posterior (López-Rodríguez et al., 2013). El objetivo del presente estudio fue determinar la influencia de la composición racial sobre variables reproductivas de calidad espermática, cinética celular y movilidad del semen en verracos.

\section{Materiales y métodos}

\section{Descripción y localización de las granjas}

Las granjas participantes en el presente estudio fueron seleccionadas con el propósito de minimizar fuentes de variación externas que, se identificaron con anterioridad al desarrollo del proyecto. En todos los casos, el mecanismo para la reproducción de los animales fue la inseminación artificial, con el equipo y personal entrenado para tal efecto. Todas las granjas fueron seleccionadas de acuerdo con un manejo reproductivo uniforme; durante la fase experimental, el ritmo de extracciones seminales (dos extracciones cada tres semanas) y descanso sexual fue similar. Asimismo, se utilizaron registros físicos y/o digitales para la trazabilidad de los eyaculados de cada animal dentro de cada granja. En el proyecto participaron un total de dieciséis granjas porcinas ubicadas en los cantones de Río Cuarto, Grecia y San Carlos de la provincia de Alajuela, y Sarapiquí en la provincia de Heredia (Cuadro 1).

Cuadro 1. Distribución de granjas porcinas participantes en el estudio de la composición racial de verracos sobre variables reproductivas fenotípicas de calidad seminal, cinética celular y movilidad del semen. Región Huetar Norte, Costa Rica. 2016.

Table 1. Participating farms distribution in the study of boar breed composition on phenotypic reproductive variables of semen quality, cells kinetics and sperm motility. North Huetar Region, Costa Rica. 2016.

\begin{tabular}{cccc}
\hline Granjas & Provincia & Cantón & Distritos \\
\hline 3 & Alajuela & Río Cuarto & Río Cuarto \\
2 & Alajuela & Grecia & Bajos del Toro \\
10 & Alajuela & San Carlos & Florencia, Pital, Pocosol, Monterrey, Aguas Zarcas \\
1 & Heredia & Sarapiquí & San Miguel \\
\hline
\end{tabular}

\section{Animales}

El estudio se realizó en 63 verracos, con edades medias comprendidas entre 23,5 y 25,3 meses (media de $24,4 \pm 10,9)$, que fueron seleccionados de las granjas participantes dentro de la región Huetar Norte, Costa Rica. Todos los animales se encontraban entrenados para la extracción seminal. Los animales se dividieron en grupos según la composición racial. Los grupos raciales se definieron por el pedigrí (en caso de apareamiento controlado dentro de la propia granja) y la procedencia si fue comprado e integrado directamente al núcleo de selección. De esta manera, se identificaron seis grupos raciales: Duroc (D), Yorkshire (Y), Landrace (L), $F_{1}$ Pietrain*Duroc (PD), línea genética comercial A (LA), que es una selección de la raza Pietrain, y línea genética comercial B (LB), que es una línea seleccionada a partir de cruzamientos controlados con la raza Pietrain. En todos los casos, se tomaron al menos dos eyaculados de cada animal (Cuadro 2). En las granjas existen verracos "abuelos" y "terminales". 
Cuadro 2. Edad promedio y desviación estándar de verracos incluidos en el estudio de la composición racial sobre variables reproductivas fenotípicas de calidad espermática, cinética celular y movilidad del semen. Región Huetar Norte, Costa Rica. 2016.

Table 2. Boar age mean and standard deviation added in the study of boar breed composition on phenotypic reproductive variables of semen quality, cells kinetics and sperm motility. North Huetar Region, Costa Rica. 2016.

\begin{tabular}{ccccc}
\hline Línea genética/raza $^{\text {a }}$ & Verracos (n) & Eyaculados (n) & Edad (meses) & SD \\
\hline D & 11 & 58 & 26,2 & 12,4 \\
Y & 10 & 27 & 24,0 & 13,9 \\
L & 6 & 31 & 27,1 & 10,7 \\
PD & 17 & 63 & 23,6 & 8,9 \\
LA & 11 & 40 & 21,5 & 6,7 \\
LB & 8 & 21 & 29,1 & 12,9 \\
\hline
\end{tabular}

${ }^{a}$ D: Duroc, L: Landrace, PD: $\mathrm{F}_{1}$-Piétrain*Duroc, $\mathrm{L}_{\mathrm{A}}$ : Línea genética A, L $\mathrm{B}_{\mathrm{B}}$ : Línea genética B, Y: Yorkshire, SD: desviación estándar / D: Duroc, L: Landrace, PD: $\mathrm{F}_{1}$-Pietrain*Duroc, LA: genetic line A, LB: genetic line B, Y: Yorkshire, SD: standard deviation.

Los primeros corresponden a animales cuya progenie se utiliza para obtener reemplazos de hembras dentro de las granjas. Los segundos son verracos cuya progenie (machos y hembras) se utiliza para enviar a mercado.

Durante el período de recolección seminal, los animales se mantuvieron en compartimentos separados (tamaño $12 \mathrm{~m}^{2}$ ), en donde se podían realizar regularmente medidas zoosanitarias como desparasitaciones. Los animales se alimentaban una vez al día, antes del momento de la recolección del semen, con 2,5 kg de un concentrado comercial para verracos (Cuadro 3). Se proporcionó agua ad libitum, mediante dispensadores automáticos.

Cuadro 3. Composición nutricional de la alimentación de verracos, empleados en el estudio de la influencia de la composición racial sobre variables reproductivas fenotípicas de calidad espermática, cinética celular y movilidad del semen. Región Huetar Norte, Costa Rica. 2016.

Table 3. Composition of feed for the boars, employed in the study of boar breed composition on phenotypic reproductive variables of semen quality, cell kinetics and sperm motility in North Huetar Region, Costa Rica. 2016.

\begin{tabular}{cc}
\hline Materia seca (\%) & $\mathbf{8 5 , 0}$ \\
\hline Proteína cruda (\%) & 18,36 \\
Extracto etéreo (\%) & 4,67 \\
Fibra cruda (\%) & 4,11 \\
Fósforo (\%) & 0,67 \\
Calcio (\%) & 0,80 \\
Sodio (\%) & 0,46 \\
Zinc (mg/kg) & 50 \\
Vitamina A (IU/kg) & 5000 \\
Vitamina D3 (IU/kg) & 625 \\
\hline
\end{tabular}

\section{Recolección de muestras de semen y procesamiento}

Se recolectaron muestras de semen fresco diluidas 1:1 (vol:vol), en un diluyente estándar comercial (BTS, Minitüb, Alemania) para preservación de semen porcino por periodo corto (tres días). Las muestras de semen se 
tomaron durante todo el año 2016. Antes de cada eyaculación, se estimuló a los verracos, llevándolos a un corral de extracción separado que contenía el potro de extracción o "maniquî́” y se obtuvo el semen mediante manipulación manual del pene después de que el verraco montara el "maniquî". Las tres últimas fracciones de semen de cada eyaculado se recogieron en recipientes graduados de colección de semen y se filtraron a través de tres capas de gasa esterilizada para separar las secreciones de glándula bulbouretral de los otros constituyentes del semen. Después de cada recolección de semen, se realizó la evaluación macroscópica del volumen. Posteriormente, se colocó la fracción filtrada en un baño maría a $37{ }^{\circ} \mathrm{C}$, donde las muestras permanecieron durante el período de envasado y determinación de la concentración.

La concentración espermática se determinó con un espectrofotómetro. El número total de espermatozoides por eyaculado por verraco, se calculó multiplicando la concentración de espermatozoides por volumen del eyaculado. Cuando hubo que recolectar muestras en más de una granja, se respetaron todos los protocolos de bioseguridad de cada granja y fueron los técnicos de cada una de ellas los que realizaban la extracción seminal. Las muestras de semen que cumplían las calidades mínimas para la IA, esto es, un mínimo de $80 \%$ de morfología normal y de concentración de espermatozoides $\left(>200 \times 10^{6}\right.$ espermatozoides $\left./ \mathrm{ml}\right)$, se colocaron en cajas de polietileno y se trasladaron al Laboratorio de Reproducción Animal del Instituto Tecnológico de Costa Rica, donde se mantuvieron almacenadas $24 \mathrm{~h}$ antes de procesarse.

\section{Análisis automatizado de movilidad y cinética espermática}

Una vez llegadas las muestras al Laboratorio de Reproducción Animal, previo a su análisis, se almacenaron en un frigorífico climatizado a $17^{\circ} \mathrm{C}$, durante $24 \mathrm{~h}$. La movilidad de las células y las características cinéticas de los espermatozoides se evaluaron con un sistema CASA (sistema integrado de análisis de semen o "Computerassisted semen analysis"), junto con un microscopio de contraste de fase (UB203i) con un objetivo de contraste de fase negativo 10x y una placa calefactada a $37^{\circ} \mathrm{C}$. Los archivos de video se grabaron y analizaron a veinticinco imágenes por segundo (fps "frame per second") durante dos segundos. La motilidad de los espermatozoides se determinó agregando una alícuota de 2,7 $\mu \mathrm{l}$ del eyaculado, previa agitación del tubo Eppendorf, en un modelo de cámara comercial ISAS-D4C20 de $20 \mu \mathrm{m}$ de profundidad. Cada muestra se analizó por duplicado y se tomaron como mínimo siete campos. Se consideró para este estudio, el área de la cabeza de los espermatozoides (área; $\mu \mathrm{m}^{2}$ ) y varios parámetros de movilidad que se describen a continuación, según clasificaciones propuestas por la Organización Mundial de la Salud (WHO, 2010).

La motilidad total (MTOT, \%), se definió como el porcentaje de células móviles que presentaron una velocidad curvilínea (VCL) $>10 \mu \mathrm{m} / \mathrm{s}$ dentro de la muestra. La motilidad progresiva (MP), indicó las células que presentaban un movimiento hacia delante en línea recta. Los mótiles no progresivos (MNP) fueron espermatozoides móviles, pero con prevalencia de movimientos circulares. La velocidad curvilínea (VCL; $\mu \mathrm{m} / \mathrm{s}$ ), se definió como la velocidad de la cabeza del espermatozoide a lo largo de la trayectoria curvilínea real. La velocidad rectilínea (VSL; $\mu \mathrm{m} / \mathrm{s}$ ) se fijó como la velocidad de la cabeza del espermatozoide a lo largo de la línea recta entre la primera y la última posición detectada. La velocidad de la trayectoria promedio (VAP; $\mu \mathrm{m} / \mathrm{s}$ ), se calculó como una interpolación entre los puntos correspondientes a la trayectoria de la VCL. El índice de rectitud (STR; \%), se definió como la linealidad de la distancia de la trayectoria promedio (VSL/VAP), y la linealidad (LIN; \%) fue la relación entre la distancia en línea recta y la distancia de la trayectoria real (VSL/VCL).

Otros parámetros cinéticos que se consideraron para caracterizar la calidad del movimiento espermático fueron el índice de oscilación (WOB = VAP/VCL; \%), la amplitud del desplazamiento lateral de la cabeza (ALH; $\mu \mathrm{m}$ ), expresado como la altura máxima (o media) de la amplitud del movimiento oscilatorio de la trayectoria curvilínea, y la frecuencia de batido (BCF; Hz), expresada como el número de veces que la trayectoria curvilínea cruza la lineal. La configuración del software se ajustó al análisis de espermatozoides del verraco: de 10 a $80 \mu \mathrm{m}^{2}$, para el 
área de la cabeza, y 11 para conectividad. Además, se determinaron los porcentajes de espermatozoides móviles progresivos, no progresivos y estáticos. El movimiento progresivo se definió como el porcentaje de espermatozoides que presentaron movimiento con un índice de rectitud (STR) $\geq 75 \%$ dentro de la muestra. Los espermatozoides estáticos correspondieron a aquellas células que presentaron una velocidad curvilínea (VCL) $<10 \mu \mathrm{m} / \mathrm{s}$. Dentro de los espermatozoides móviles, se determinó el porcentaje de células con movimiento considerado rápido, medio y lento, de acuerdo con el criterio de velocidad $(\mu \mathrm{m} / \mathrm{s}): 10<$ lento $25<$ medio $<45$ rápido.

\section{Análisis estadístico}

Se realizaron pruebas de normalidad y homocedasticidad, usando Shapiro-Wilk y Levene, respectivamente, a los datos obtenidos del análisis de todos los parámetros espermáticos. Al intentar ajustar al modelo de la distribución normal, algunas variables se transformaron usando la función arcoseno de la raíz cuadrada $(\arcsin \sqrt{ } \mathrm{x})$ antes de ejecutar el ANOVA de medidas repetidas. Incluso, después de la transformación arcoseno cuando las variables no se distribuyeron con arreglo a la normal, se realizaron análisis no paramétricos con la prueba de Kruskal-Wallis, y cuando se detectaron diferencias estadísticamente significativas al usar esta prueba, se utilizó la prueba U no paramétrica de Mann-Whitney para comparar pares de valores directamente. Cuando se comprobaron las hipótesis de la distribución normal y la homogeneidad de varianzas, se realizó un ANOVA de medidas repetidas. El modelo estadístico utilizado fue del tipo:

$Y_{i j k l m n o}=\mu+\mathrm{G}_{i}+E_{j}+R_{k}+A_{L}+E Y_{M(L)}+E D_{n}+(E E D)_{j n}+(E R)_{j k}+(R E D)_{k n}+\varepsilon_{(i j k l m n) o}$

Donde,

$Y_{i j k l m n o}=o$-ésima observación correspondiente al $i$-ésimo factor $\mathrm{G}$, del $j$-ésimo factor $\mathrm{E}$, en el $k$-ésimo factor R, del $l$-ésimo del factor A en el nivel $m$-ésimo del factor EY dentro del nivel $l$-ésimo del factor A del nivel $m$-ésimo del factor ED (observación= $1,2, \ldots, o$ ).

$\mu=$ media general.

$G_{i}=$ efecto del nivel $i$-ésimo del factor $\mathrm{G}$ (granja; $i=1,2,3, \ldots, g$ ).

$E_{j}=$ efecto del nivel $j$-ésimo del factor $E$ (época; $j=1,2$ ).

$R_{K}=$ efecto del nivel $k$-ésimo del factor $\mathrm{R}$ (grupo racial; $k=1,2,3, \ldots, r$ )

$A_{L}=$ efecto del nivel $l$-ésimo del factor A (animal; $l=1,2,3, \ldots, a$ )

$E Y_{M(L)}=$ efecto producido por el nivel $m$-ésimo del factor EY dentro del nivel $l$-ésimo del factor A (eyaculado; $m=1,2,3, \ldots, e y)$

$E D_{N}=$ efecto del nivel n-ésimo del factor ED (edad; $\left.\mathrm{n}=1,2,3\right)$

$(E E D)_{j n}=$ efecto producido por la interacción $\mathrm{E}^{*} \mathrm{ED}$.

$(E R)_{j k}=$ efecto producido por la interacción $\mathrm{E}^{*} \mathrm{R}$

$(R E D)_{k n}=$ efecto producido por la interacción $\mathrm{R} * \mathrm{ED}$

$\varepsilon_{(i j k l m n) o}=$ error experimental. Se suponen variables aleatorias independientes $N(0, \sigma)$

Los modelos lineales generales y mixtos se utilizaron para analizar las variables de cinética y movilidad del semen de verracos de diferentes razas o composición genética híbrida. El espacio muestral $(\Omega)$ total fue de 63 unidades experimentales. Se realizaron pruebas de comparación múltiple por el método de mínimos cuadrados, y se utilizó la corrección de Bonferroni, con un nivel de significación estadística de $\leq 0,05$.

Se realizó análisis multivariante con el propósito de identificar subpoblaciones de espermatozoides del conjunto completo de datos de movilidad. El primer paso fue realizar un análisis de componentes principales 
(PCA). Para seleccionar el número de componentes principales a utilizar en el próximo paso del análisis, se siguió el criterio de seleccionar solo aquellos con un valor propio (varianza extraída para ese componente principal particular) $>1$ (criterio de Kaiser). Además, se comprobó la factibilidad del análisis factorial (AF), mediante la prueba de esfericidad de Bartlett para contrastar la hipótesis nula, de que la matriz de correlaciones es una matriz identidad, y el índice KMO (Kaiser-Meyer-Olkin), determina el cálculo de las correlaciones entre dos variables una vez eliminada la influencia que las restantes variables ejercen sobre ellas e indica la conveniencia de realizar el AF.

El segundo paso fue realizar un procedimiento de clúster de dos pasos con los índices derivados obtenidos después del PCA con los datos seminales. Todas las mediciones de los espermatozoides dentro de cada granja, raza, animal y eyaculado, se agruparon mediante parámetros de forma y tamaño, con un procedimiento de agrupamiento no jerárquico (modelo k-medias y distancia euclídea). Esto clasifica los espermatozoides del conjunto de datos en un pequeño número de subpoblaciones de acuerdo con la velocidad, linealidad y progresividad de las células. Este análisis permitió la identificación de subpoblaciones de espermatozoides y la detección de valores atípicos.

Los análisis estadísticos se realizaron usando Statgraphics Centurion XVII, 17.2.04. (32-bit) (1982-2016 por Statpoint Technologies Inc., USA).

\section{Resultados}

\section{Análisis convencional del semen}

Se encontró un efecto $(\mathrm{P}<0,05)$ de granja para las variables: concentración del eyaculado, volumen, número total de espermatozoides y motilidad total. El efecto de eyaculado también fue significativo para las variables anteriores, excepto para el volumen de eyaculado. La época no fue significativa $(\mathrm{P}>0,05)$ para las variables analizadas. Los efectos del grupo racial y las interacciones época* grupo racial y edad*grupo racial, fueron significativos $(\mathrm{P}<0,05)$ para las variables de movilidad estudiadas, excepto para la concentración de espermatozoides.

Los resultados de la motilidad total, la concentración espermática, el volumen del eyaculado y el número total de espermatozoides por eyaculado de los verracos de las diferentes razas o líneas genéticas, se muestran en la Figura 1. La motilidad total fue estadísticamente diferente $(\mathrm{P}<0,05)$ entre los grupos raciales Landrace y $\mathrm{F}_{1}$ Pietrain*Duroc. El volumen de eyaculado fue la variable que mostró más diferencia entre grupos $(\mathrm{P}<0,05)$, especialmente entre los verracos de las razas Yorkshire, línea A, línea B y Landrace, en orden decreciente. Además, el análisis estadístico indicó que, no había diferencias significativas en la concentración de espermatozoides entre los verracos de diferentes grupos raciales $(\mathrm{P}>0,05)$. Los verracos de la raza Duroc presentaron un menor número total de espermatozoides con respecto a los demás grupos raciales, mientras que, los verracos de la raza Yorkshire presentaron mayor número total de espermatoides en relación con los verracos de la Línea $\mathrm{B}$ y los machos $\mathrm{F}_{1}$ Pietrain*Duroc $(\mathrm{P}<0,05$; Figura 1).

\section{Análisis automatizado del semen por un sistema CASA-Mot}

El efecto de granja fue significativo $(\mathrm{P}<0,05)$ para todas las variables de cinética. El grupo racial también resultó significativo para las variables de cinética, excepto para ALH. Los efectos de eyaculado, época y edad, no resultaron significativos $(\mathrm{P}>0,05)$ para las variables de cinética, excepto para VAP $(\mathrm{P}<0,05)$. La interacción época*grupo racial mostró un efecto significativo $(\mathrm{P}<0,05)$ para velocidad rectilínea y linealidad. La interacción edad*grupo racial solo fue significativa para las velocidades $(\mathrm{P}<0,05)$. La interacción época*edad no fue significativo $(\mathrm{P}>0,05)$.

Se encontró un efecto $(\mathrm{P}<0,05)$ del grupo racial en los parámetros de cinética espermática, excepto para la ALH. La VCL fue estadísticamente diferente entre la línea genética A y la raza Yorkshire, con una diferencia de 

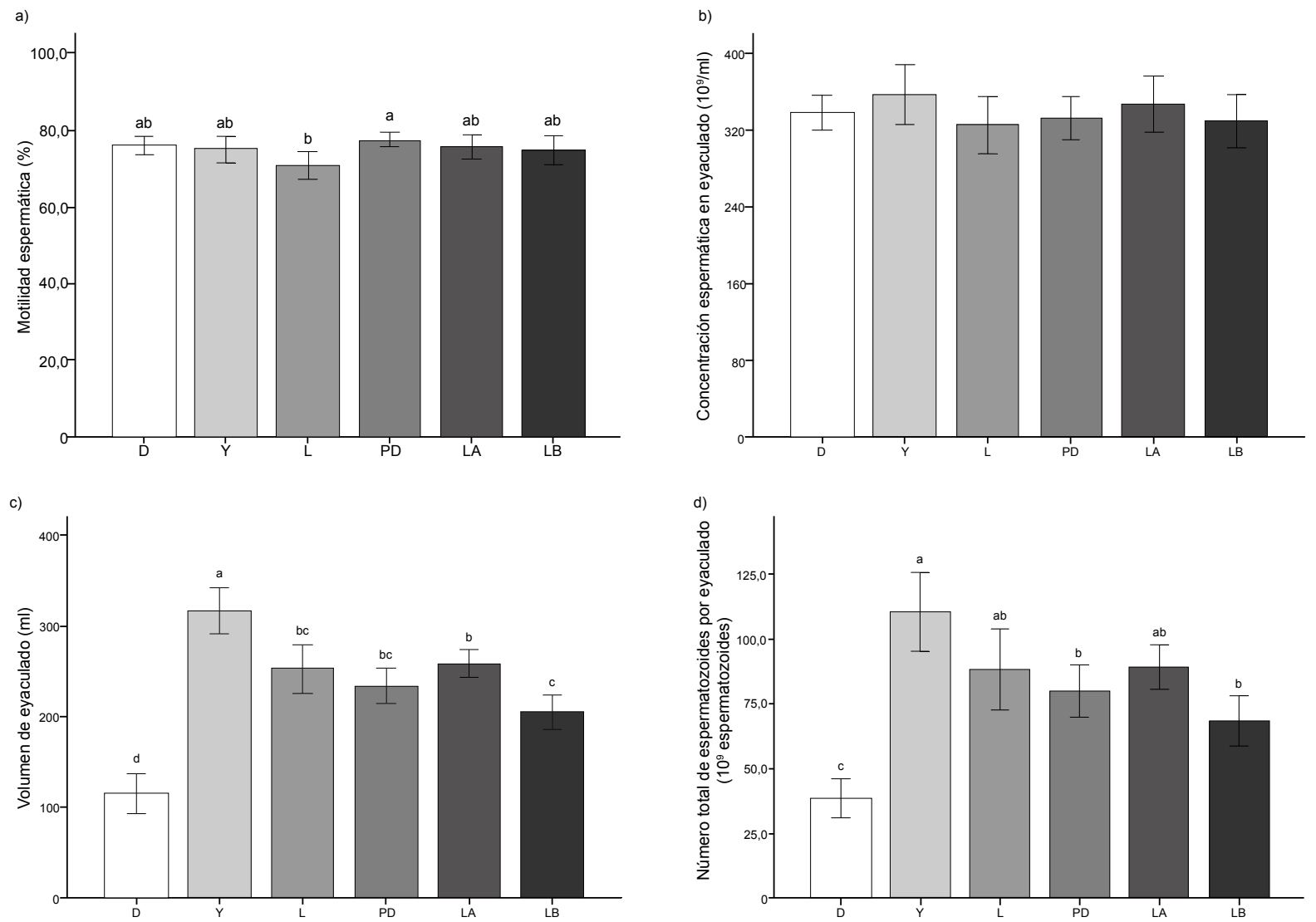

Figura 1. (a) Motilidad espermática, (b) concentración de espermatozoides en eyaculado, (c) volumen de eyaculado, (d) número total de espermatozoides en eyaculado por raza o línea genética de los verracos empleados en el estudio de la influencia de la composición racial sobre variables reproductivas fenotípicas de calidad espermática, cinética celular y movilidad del semen. Región Huetar Norte, Costa Rica. 2016.

D: Duroc, L: Landrace, PD: F1-Piétrain*Duroc, LA: línea genética A, LB: línea genética B, Y: Yorkshire.

Resultados se expresan como medias mínimo-cuadráticas con intervalos de confianza al 95\% (IC95\%). ${ }^{\text {a,b,c,d }}$ Valores con distinto superíndice difieren significativamente $\mathrm{P}<0,05$.

Figure 1. (a) Sperm motility, (b) concentration of spermatozoa in ejaculate, (c) ejaculate volume, (d) total number of spermatozoa per ejaculate per boar breeds or genetic line added in the breed composition study on phenotypic reproductive variables of semen quality, cells kinetics and sperm motility. North Huetar Region, Costa Rica. 2016.

D: Duroc, L: Landrace, PD: F1-Pietrain*Duroc, LA: genetic line A, LB: genetic line B, Y: Yorkshire.

Results are expressed as least square mean and 95\% confidence interval of the mean (95\%CI). ${ }^{\mathrm{a}, \mathrm{b}, \mathrm{c}, \mathrm{d}}$ Values with different superscripts differ $\mathrm{P}<0.05$.

medias de $5,63 \pm 1,86 \mu \mathrm{m} / \mathrm{s}$. La línea genética A, también presentó los mayores valores $(\mathrm{P}<0,05)$ de VSL y VAP. La VSL fue diferente entre esta línea genética con respecto a la raza Landrace y el $\mathrm{F}_{1}$ Pietrain*Duroc, y no presentó diferencias entre estos dos últimos grupos raciales, mientras que, para la VAP, hubo diferencias entre la línea A y la raza Landrace, el $\mathrm{F}_{1}$ Pietrain*Duroc y la línea genética $\mathrm{B}$. La raza que presentó el mayor índice de linealidad (LIN) fue la línea genética $B$, mientras que, el menor fue mostrado por la $F_{1}$ Pietrain*Duroc. No hubo diferencias entre el STR de las dos líneas genéticas (A y B), mientras que, para el WOB, las diferencias estadísticas detectadas fueron entre la línea $\mathrm{B}$ y el $\mathrm{F}_{1}$ Pietrain*Duroc, este último presentó el menor índice de oscilación. Para la BCF solo hubo diferencias entre las líneas genéticas A y B (Cuadro 4). 
Cuadro 4. Parámetros cinéticos de calidad espermática (medias $\pm E E$ ) de verracos según línea genética y/o raza, empleados en el estudio de la influencia de la composición racial sobre variables reproductivas fenotípicas de calidad espermática, cinética celular y movilidad del semen, en la Región Huetar Norte, Costa Rica. 2016.

Table 4. Kinematic parameters of boar sperm quality $(\mathrm{LSM} \pm \mathrm{SE})$ for genetic line and/or breed added in the breed composition study on phenotypic reproductive variables of semen quality, cells kinetics and sperm motility. North Huetar Region, Costa Rica. 2016.

\begin{tabular}{lcccccc}
\hline & \multicolumn{5}{c}{ Grupo racial* } \\
\cline { 2 - 7 } Parámetro cinético & D & Y & L & PD & LA & LB \\
\hline VCL $(\mu \mathrm{m} / \mathrm{s})$ & $58,91 \pm 1,01^{\text {ab }}$ & $55,08 \pm 1,42^{\mathrm{b}}$ & $57,20 \pm 1,30^{\mathrm{ab}}$ & $57,75 \pm 0,90^{\mathrm{ab}}$ & $60,71 \pm 1,12^{\mathrm{a}}$ & $54,02 \pm 1,53^{\mathrm{b}}$ \\
VSL $(\mu \mathrm{m} / \mathrm{s})$ & $29,70 \pm 0,67^{\mathrm{ab}}$ & $29,05 \pm 0,90^{\mathrm{ab}}$ & $27,45 \pm 0,86^{\mathrm{b}}$ & $27,24 \pm 0,60^{\mathrm{b}}$ & $31,19 \pm 0,75^{\mathrm{a}}$ & $29,59 \pm 0,97^{\mathrm{ab}}$ \\
VAP $(\mu \mathrm{m} / \mathrm{s})$ & $38,08 \pm 0,66^{\mathrm{ab}}$ & $37,16 \pm 1,0^{\mathrm{ab}}$ & $36,28 \pm 0,85^{\mathrm{b}}$ & $35,99 \pm 0,59^{\mathrm{b}}$ & $40,02 \pm 0,74^{\mathrm{a}}$ & $36,62 \pm 0,97^{\mathrm{b}}$ \\
LIN $(\%)$ & $51,16 \pm 1,09^{\mathrm{ab}}$ & $52,69 \pm 1,52^{\mathrm{ab}}$ & $49,16 \pm 1,40^{\mathrm{ab}}$ & $48,08 \pm 0,98^{\mathrm{b}}$ & $51,67 \pm 1,23^{\mathrm{ab}}$ & $55,55 \pm 1,64^{\mathrm{a}}$ \\
STR $(\%)$ & $77,41 \pm 0,91^{\mathrm{a}}$ & $77,43 \pm 1,27^{\mathrm{a}}$ & $75,04 \pm 1,16^{\mathrm{b}}$ & $75,08 \pm 0,81^{\mathrm{b}}$ & $77,64 \pm 1,02^{\mathrm{a}}$ & $80,25 \pm 1,37^{\mathrm{a}}$ \\
WOB $(\%)$ & $65,47 \pm 0,83^{\mathrm{ab}}$ & $67,51 \pm 1,17^{\mathrm{a}}$ & $64,38 \pm 1,07^{\mathrm{ab}}$ & $63,11 \pm 0,75^{\mathrm{b}}$ & $66,21 \pm 0,94^{\mathrm{ab}}$ & $68,43 \pm 1,26^{\mathrm{a}}$ \\
ALH $(\mu \mathrm{m})$ & $2,61 \pm 0,11$ & $2,26 \pm 0,16$ & $2,33 \pm 0,15$ & $2,39 \pm 0,10$ & $2,41 \pm 0,13$ & $2,15 \pm 0,18$ \\
BCF $(\mathrm{Hz})$ & $7,91 \pm 0,08^{\mathrm{ab}}$ & $7,62 \pm 0,11^{\mathrm{ab}}$ & $7,76 \pm 0,10^{\mathrm{ab}}$ & $7,65 \pm 0,07^{\mathrm{ab}}$ & $8,00 \pm 0,09^{\mathrm{a}}$ & $7,55 \pm 0,12^{\mathrm{b}}$ \\
\hline
\end{tabular}

* D: Duroc, L: Landrace, PD: F1-Piétrain*Duroc, LA: Línea genética A, LB: Línea genética B, Y: Yorkshire. VCL: velocidad curvilínea, $\mu \mathrm{m} / \mathrm{s}$; VSL: velocidad rectilínea, $\mu \mathrm{m} / \mathrm{s}$; VAP: velocidad media, $\mu \mathrm{m} / \mathrm{s}$; LIN: índice de linealidad, \%; STR: índice de rectitud, \%; WOB: índice de oscilación, \%; ALH: desplazamiento lateral de la cabeza, $\mu \mathrm{m}$; BCF: frecuencia de entrecruzamiento, Hz. EE: error estándar. ${ }^{a, b}$ Dentro de fila, valores con diferente superíndice presentan diferencias significativas $\mathrm{P}<0,05$ / *D: Duroc, L: Landrace, PD: F1-Pietrain*Duroc, LA: genetic line A, LB: genetic line B, Y: Yorkshire. VCL: curvilinear velocity, $\mu \mathrm{m} / \mathrm{s}$; VSL: straight line velocity, $\mu \mathrm{m} / \mathrm{s}$; VAP: average path velocity, $\mu \mathrm{m} / \mathrm{s}$; LIN: linearity of forward progression, \%; STR: straightness, \%; WOB: wobble, \%; ALH: amplitude of lateral head displacement, $\mu \mathrm{m}$; BCF: beat-cross frequency, Hz; LSM: Least squares means; SE: standard error. ${ }^{\mathrm{a}, \mathrm{b}}$ Within the same row, values with different superscripts show significant difference at $\mathrm{P}<0.05$.

Los efectos de granja, eyaculado, época y grupo racial, así como los de las interacciones época*grupo racial y edad*grupo racial, resultaron significativos $(\mathrm{P}<0,05)$ para los espermatozoides móviles progresivos, móviles no progresivos y rápidos. Para los espermatozoides clasificados como medios, todos los efectos resultaron significativos excepto la época. En los espermatozoides clasificados como lentos, solo el eyaculado y la edad no resultaron significativos $(\mathrm{P}>0,05)$. La granja, el eyaculado, el grupo racial y las interacciones época*grupo racial y edad*grupo racial, fueron significativos $(\mathrm{P}<0,05)$ para los espermatozoides clasificados como estáticos.

La categorización de los espermatozoides según su velocidad en rápidos ( $>45 \mu \mathrm{m} / \mathrm{s})$, medios $(25 \leq x<45 \mu \mathrm{m} / \mathrm{s})$, lentos $(10<\mathrm{y}<25 \mu \mathrm{m} / \mathrm{s})$ y estáticos, mostró para la categoría de movimiento rápido diferencias entre las razas Duroc, Yorkshire y Landrace, con diferencias entre medias de 7,48, 11,68 y 4,20\%, respectivamente. No hubo diferencias $(P>0,05)$ entre la raza Duroc y la línea B, ni entre la raza Yorkshire y la línea A.

Las razas con mayores porcentajes de espermatozoides lentos fueron Yorkshire y Landrace, con valores de 11,49 y $10,11 \%$. No hubo diferencias $(\mathrm{P}>0,05)$ entre el porcentaje de espermatozoides lentos en la raza Duroc, $\mathrm{F}_{1}$ Pietrain*Duroc, línea A y línea B. La raza Landrace presentó el mayor porcentaje de espermatozoides estáticos $(29,30 \% ; \mathrm{P}<0,05)$ con respecto a los demás grupos raciales (Cuadro 5).

Los grupos raciales que más se diferenciaron $(\mathrm{P}<0,05)$ en los valores de motilidad total y progresiva fueron $\mathrm{F}_{1}$ Pietrain*Duroc y Landrace, con una diferencia de medias de 6,77\% y $8,05 \%$, respectivamente, mientras que, para los demás grupos raciales no hubo diferencias $(\mathrm{P}>0,05)$. La línea genética $\mathrm{B}$ presentó los valores menores de espermatozoides mótiles no progresivos con un valor de 16,50\%, mientras que, para los demás grupos raciales no se presentaron diferencias $(\mathrm{P}>0,05)$. La línea genética A presentó el mayor valor $(\mathrm{P}<0,05)$ de espermatozoides 
Cuadro 5. Porcentajes (medias $\pm \mathrm{EE}$ ) de espermatozoides de verraco con movimiento categorizado como rápido $(>45 \mu \mathrm{m} / \mathrm{s})$, medio $(25 \leq x<45 \mu \mathrm{m} / \mathrm{s})$ y lento $(10<\mathrm{y}<25 \mu \mathrm{m} / \mathrm{s})$, así como espermatozoides estáticos, según grupo racial o línea genética. Región Huetar Norte, Costa Rica. 2016.

Table 5. Percentages (LSM \pm SE) of boar spermatozoa with movement rapid $(>45 \mu \mathrm{m} / \mathrm{s})$, medium $(25 \leq \mathrm{x}<45 \mu \mathrm{m} / \mathrm{s})$ and slow $(10<\mathrm{y}<25 \mu \mathrm{m} / \mathrm{s})$, as well as static spermatozoa for breed, according to racial group or genetic line. North Huetar Region of Costa Rica. 2016.

\begin{tabular}{|c|c|c|c|c|c|c|}
\hline & \multicolumn{6}{|c|}{ Grupo racial* } \\
\hline & D & $\mathbf{Y}$ & $\mathbf{L}$ & PD & LA & LB \\
\hline Rápidos & $48,21 \pm 2,10^{\mathrm{a}}$ & $40,73 \pm 2,37^{b}$ & $36,53 \pm 2,18^{c}$ & $47,34 \pm 1,51^{\mathrm{a}}$ & $42,86 \pm 1,90^{\mathrm{b}}$ & $46,68 \pm 2,56^{\mathrm{a}}$ \\
\hline Medios & $19,61 \pm 1,08^{b}$ & $22,71 \pm 1,22^{\mathrm{ab}}$ & $24,12 \pm 1,12^{\mathrm{a}}$ & $22,82 \pm 0,77^{\mathrm{ab}}$ & $23,65 \pm 0,98^{\mathrm{a}}$ & $19,99 \pm 1,31^{\mathrm{b}}$ \\
\hline Lentos & $8,96 \pm 0,83^{\mathrm{bc}}$ & $11,49 \pm 0,94^{\mathrm{a}}$ & $10,11 \pm 0,86^{\mathrm{ab}}$ & $7,42 \pm 0,59^{\mathrm{c}}$ & $8,94 \pm 0,75^{\mathrm{bc}}$ & $7,96 \pm 1,01^{\mathrm{bc}}$ \\
\hline Estáticos & $24,36 \pm 1,52^{b}$ & $25,08 \pm 1,72^{b}$ & $29,30 \pm 1,57^{\mathrm{a}}$ & $22,43 \pm 1,09^{b}$ & $24,58 \pm 1,37^{b}$ & $25,40 \pm 1,85^{\mathrm{b}}$ \\
\hline
\end{tabular}

* D: Duroc, L: Landrace, PD: F1-Piétrain*Duroc, LA: línea genética A, LB: línea genética B, Y: Yorkshire. EE: error estándar. ${ }^{a, b, c}$ Dentro de fila, valores con diferente superíndice presentan diferencias significativas P $<0,05$ / * D: Duroc, L: Landrace, PD: F1Pietrain*Duroc, LA: genetic line A, LB: genetic line B, Y: Yorkshire. LSM: Least squares means; SE: standard error. ${ }^{\mathrm{a}, \mathrm{b}, \mathrm{c}}$ Within the same row, values with different superscripts show significant difference at $\mathrm{P}<0.05$.

mótiles progresivos rápidos; para los demás grupos raciales no hubo diferencias significativas y el rango de valores varió entre 41,30 y $44,01 \%$ (Cuadro 6).

Cuadro 6. Porcentajes (medias \pm EE) de espermatozoides de verraco con movilidad progresiva según clasificación de la WHO (2010), según grupo racial o línea genética. Región Huetar Norte, Costa Rica. 2016.

Table 6. Percentages (LSM \pm SE) of boar spermatozoa with progressive motility, as classified by WHO (2010) for breed, according to racial group or genetic line. North Huetar Region, Costa Rica. 2016.

\begin{tabular}{lcccccc}
\hline & \multicolumn{6}{c}{ Grupo racial* $^{*}$} \\
\cline { 2 - 7 } & D & Y & L & PD & LA & LB \\
\hline MTOT & $75,82 \pm 1,52^{\mathrm{ab}}$ & $74,93 \pm 1,71^{\mathrm{ab}}$ & $70,71 \pm 1,57^{\mathrm{b}}$ & $77,48 \pm 1,09^{\mathrm{a}}$ & $75,44 \pm 1,37^{\mathrm{ab}}$ & $74,62 \pm 1,85^{\mathrm{ab}}$ \\
MP & $53,70 \pm 1,90^{\mathrm{ab}}$ & $54,08 \pm 2,15^{\mathrm{ab}}$ & $51,80 \pm 1,97^{\mathrm{b}}$ & $59,85 \pm 1,37^{\mathrm{a}}$ & $56,14 \pm 1,72^{\mathrm{ab}}$ & $58,13 \pm 2,32^{\mathrm{ab}}$ \\
MNP & $20,90 \pm 1,28^{\mathrm{a}}$ & $20,86 \pm 1,45^{\mathrm{a}}$ & $18,91 \pm 1,33^{\mathrm{a}}$ & $17,72 \pm 0,92^{\mathrm{a}}$ & $19,31 \pm 1,16^{\mathrm{a}}$ & $16,50 \pm 1,57^{\mathrm{b}}$ \\
MPR & $42,98 \pm 2,12^{\mathrm{b}}$ & $41,30 \pm 2,40^{\mathrm{b}}$ & $42,85 \pm 2,20^{\mathrm{b}}$ & $43,56 \pm 1,53^{\mathrm{b}}$ & $50,03 \pm 1,92^{\mathrm{a}}$ & $44,01 \pm 2,59^{\mathrm{b}}$ \\
\hline
\end{tabular}

* D: Duroc, L: Landrace, PD: F1-Piétrain*Duroc, LA: línea genética A, LB: línea genética B, Y: Yorkshire. EE: error estándar. MTOT: motilidad total, MP: motilidad progresiva $(>5 \mu \mathrm{m} / \mathrm{s})$, MNP: motilidad no progresiva $(<5 \mu \mathrm{m} / \mathrm{s})$, MPR: motilidad progresiva rápida $(\geq 25 \mu \mathrm{m} / \mathrm{s})$. $^{\mathrm{a}, \mathrm{b}}$ Dentro de fila, valores con diferente superíndice presentan diferencias significativas $\mathrm{P}<0,05 / * \mathrm{D}$ : Duroc, L: Landrace, PD: F1-Pietrain*Duroc, LA: genetic line A, LB: genetic line B, Y: Yorkshire. LSM: Least squares means; SE: standard error. MTOT: total motility, MP: progressive motility $(>5 \mu \mathrm{m} / \mathrm{s})$, MNP: non-progressive motility $(<5 \mu \mathrm{m} / \mathrm{s})$, MPR: rapid progressive motility $(\geq 25$ $\mu \mathrm{m} / \mathrm{s})$. ${ }^{\mathrm{a}, \mathrm{b}}$ Within the same row, values with different superscripts show significant difference at $\mathrm{P}<0.05$.

Hubo un efecto $(\mathrm{P}<0,05)$ del tipo de verraco sobre la movilidad espermática en variables como la motilidad total, la motilidad progresiva y sobre la clasificación de espermatozoides en rápidos, medios, lentos y estáticos.

Aunque hubo un efecto $(\mathrm{P}<0,05)$ del tipo de verraco sobre la motilidad progresiva, las diferencias no fueron biológicamente relevantes. Los verracos "abuelos" presentaron células menos rápidas en relación con los 
verracos "terminales" (36,26 vs 45,60, respectivamente). Además, los "abuelos" presentaron mayor cantidad de espermatozoides medios, lentos y estáticos, con diferencias de medias con respecto a los "terminales" de 2,22, 2,75 y 4,39\%, respectivamente. El efecto del tipo de verraco no influyó ( $P>0,05)$ sobre variables como la motilidad no progresiva y la motilidad progresiva de espermatozoides clasificados como rápidos (Cuadro 7).

Cuadro 7. Parámetros de calidad del movimiento (medias \pm EE) de espermatozoides de verraco según la función reproductiva dentro de granja. Región Huetar Norte, Costa Rica. 2016.

Table 7. Boar sperm motility quality parameters ( $\mathrm{LSM} \pm \mathrm{SE}$ ) according to reproductive function into the farm. North Huetar Region, Costa Rica. 2016.

\begin{tabular}{|c|c|c|}
\hline \multirow[b]{2}{*}{ Parámetro (\%) } & \multicolumn{2}{|c|}{ Tipo de verraco* } \\
\hline & Terminal & Abuelo \\
\hline MTOT & $75,74 \pm 0,62^{a}$ & $71,36 \pm 1,63^{b}$ \\
\hline MP & $56,75 \pm 0,77^{\mathrm{a}}$ & $52,32 \pm 2,01^{\mathrm{a}}$ \\
\hline MNP & $19,02 \pm 0,51$ & $19,05 \pm 1,33$ \\
\hline MPR & $44,16 \pm 0,88$ & $45,30 \pm 2,30$ \\
\hline Rápidos & $45,60 \pm 0,86^{\mathrm{a}}$ & $36,26 \pm 2,23^{b}$ \\
\hline Medios & $21,72 \pm 0,44^{\mathrm{a}}$ & $23,94 \pm 1,15^{\mathrm{a}}$ \\
\hline Lentos & $8,46 \pm 0,34^{\mathrm{a}}$ & $11,21 \pm 0,88^{\mathrm{b}}$ \\
\hline Estáticos & $24,26 \pm 0,63^{\mathrm{a}}$ & $28,65 \pm 1,62^{b}$ \\
\hline
\end{tabular}

* Terminal: verraco cuya progenie es enviada a mercado, Abuelo: verraco cuya progenie se utiliza para obtener reemplazos dentro de la granja. MTOT: motilidad total, MP: motilidad progresiva $(>5 \mu \mathrm{m} / \mathrm{s})$, MNP: motilidad no progresiva $(<5 \mu \mathrm{m} / \mathrm{s})$, MPR: motilidad progresiva rápida $(\geq 25 \mu \mathrm{m} / \mathrm{s})$. EE: error estándar. Rápidos $(>45 \mu \mathrm{m} / \mathrm{s})$, medios $(25 \leq \mathrm{x}<45 \mu \mathrm{m} / \mathrm{s})$, lentos $(10<\mathrm{y}<25 \mu \mathrm{m} / \mathrm{s})$. ${ }^{\mathrm{a}, \mathrm{b}}$ Dentro de fila, valores con diferente superíndice presentan diferencias significativas $\mathrm{P}<0,05 / *$ Terminal: boar whose offspring sent to market, Abuelo: boar whose offspring is then used to access replacements into the farm. MTOT: total motility, MP: progressive motility (> $5 \mu \mathrm{m} / \mathrm{s})$, MNP: non-progressive motility $(<5 \mu \mathrm{m} / \mathrm{s})$, MPR: rapid progressive motility $(\geq 25 \mu \mathrm{m} / \mathrm{s})$. LSM: Least squares means; SE: standard error. Rapid $(>45 \mu \mathrm{m} / \mathrm{s})$, medium $(25 \leq \mathrm{x}<45 \mu \mathrm{m} / \mathrm{s})$, slow $(10<\mathrm{y}<25 \mu \mathrm{m} / \mathrm{s})$. ${ }^{\mathrm{a}, \mathrm{b}}$ Within the same row, values with different superscripts show significant difference at $\mathrm{P}<0.05$.

La tendencia en los verracos adultos fue a presentar mayores volúmenes de eyaculado, y como consecuencia mayor número total de espermatozoides. La tendencia fue similar para las variables de cinética en donde los verracos adultos tendieron a presentar mayores velocidades (VSL y VAP) que los verracos jóvenes. La edad de los animales no fue relevante $(\mathrm{P}>0,05)$ para los índices cinéticos (Cuadro 8).

El análisis de componentes principales permitió extraer cuatro componentes según el criterio Kaiser $>1$, y explicó $87,47 \%$ de la varianza total. El primer componente se caracterizó por presentar células "lineales", es decir, con índices de rectitud y linealidad. El segundo correspondió a células caracterizadas por su "velocidad" curvilínea, promedio y lineal. El tercer componente principal se asoció a células con un "movimiento rápido y progresivo", mientras que, el cuarto estuvo relacionado con "células no deseadas", categorizado por espermatozoides con movimiento no progresivo y lento (Cuadro 9). En el análisis de subpoblaciones por medio de "clusters" multivariante realizado sobre 10420 espermatozoides se identificaron cuatro subpoblaciones espermáticas con distintos tipos de movimiento. 
Cuadro 8. Parámetros espermáticos y cinéticos (medias \pm DE) según edad del verraco, empleado en el estudio de la influencia de la composición racial sobre variables reproductivas fenotípicas de calidad espermática, cinética celular y movilidad del semen. Región Huetar Norte, Costa Rica. 2016.

Table 8. Sperm and kinematic parameters of boar $(\mathrm{LSM} \pm \mathrm{SD})$ for age intervals used in the breed composition study on phenotypic reproductive variables of semen quality, cells kinetics and sperm motility. North Huetar Region, Costa Rica. 2016.

\begin{tabular}{lccc}
\hline Variable & \multicolumn{2}{c}{ Edad (meses) } & $\geq \mathbf{1 8}$ \\
\cline { 2 - 4 } & $\mathbf{<}$ & $\mathbf{8} \mathbf{8} \mathbf{\mathbf { 1 8 }}$ & $75,08 \pm 0,74$ \\
\hline MTOT $(\%)$ & $76,53 \pm 2,63$ & $75,17 \pm 1,04$ & $0,34 \pm 0,01$ \\
CONC $\left(10^{9} / \mathrm{ml}\right)$ & $0,34 \pm 0,02$ & $0,33 \pm 0,01$ & $246,41 \pm 6,49^{\mathrm{a}}$ \\
VOL $(\mathrm{ml})$ & $212,88 \pm 19,17^{\mathrm{ab}}$ & $207,76 \pm 8,64^{\mathrm{b}}$ & $87,83 \pm 3,10^{\mathrm{a}}$ \\
NTEE $\left(10^{9}\right)$ & $73,60 \pm 9,15^{\mathrm{ab}}$ & $61,95 \pm 4,01^{\mathrm{b}}$ & $56,62 \pm 0,91$ \\
MP $(\%)$ & $59,44 \pm 3,24$ & $54,81 \pm 1,28$ & $18,46 \pm 0,60$ \\
\hline MNP $(\%)$ & $17,12 \pm 2,14$ & $20,45 \pm 0,84$ & $57,34 \pm 0,60^{\mathrm{b}}$ \\
VCL $(\mu \mathrm{m} / \mathrm{s})$ & $54,22 \pm 2,15^{\mathrm{b}}$ & $59,49 \pm 0,85^{\mathrm{a}}$ & $28,98 \pm 0,40^{\mathrm{a}}$ \\
VSL $(\mu \mathrm{m} / \mathrm{s})$ & $25,94 \pm 1,42^{\mathrm{b}}$ & $29,21 \pm 0,56^{\mathrm{a}}$ & $37,22 \pm 0,39^{\mathrm{a}}$ \\
VAP $(\mu \mathrm{m} / \mathrm{s})$ & $34,27 \pm 1,41^{\mathrm{b}}$ & $38,18 \pm 0,56^{\mathrm{a}}$ & $51,24 \pm 0,64$ \\
LIN $(\%)$ & $47,87 \pm 2,29$ & $49,87 \pm 0,91$ & $77,43 \pm 0,53$ \\
STR $(\%)$ & $74,46 \pm 1,89$ & $75,76 \pm 0,75$ & $65,52 \pm 0,49$ \\
WOB $(\%)$ & $63,38 \pm 1,76$ & $64,94 \pm 0,70$ & $2,34 \pm 0,07$ \\
ALH $(\mu \mathrm{m})$ & $2,33 \pm 0,23$ & $2,53 \pm 0,09$ & $7,70 \pm 0,05^{\mathrm{b}}$ \\
BCF $(\mathrm{Hz})$ & $7,47 \pm 0,17^{\mathrm{b}}$ & $7,93 \pm 0,07^{\mathrm{a}}$ & \\
\hline
\end{tabular}

MTOT: motilidad espermática, CONC: concentración de espermatozoides en eyaculado, VOL: volumen de eyaculado, NTEE: número total de espermatozoides en eyaculado. VCL: velocidad curvilínea; VSL: velocidad rectilínea; VAP: velocidad media; LIN: índice de linearidad; STR: índice de rectitud; WOB: índice de oscilación; ALH: desplazamiento lateral de la cabeza; BCF: frecuencia de entrecruzamiento. EE: error estándar. ${ }^{\mathrm{a}, \mathrm{b}}$ Dentro de fila, valores con diferente superíndice presentan diferencias significativas $\mathrm{P}<0,05$ / MTOT: total motility of spermatozoa, CONC: concentration of spermatozoa in ejaculate, VOL: ejaculate volume, NTEE: total number of spermatozoa per ejaculate. VCL: curvilinear velocity; VSL: straight line velocity; VAP: average path velocity; LIN: linearity of forward progression; STR: straightness; WOB: wobble; ALH: amplitude of lateral head displacement; BCF: beat-cross frequency; LSM: Least squares mean; SE: standard error. ${ }^{a, b}$ Within the same row, values with different superscripts show significant difference at $\mathrm{P}<0.05$.

La subpoblación 1: constituida por espermatozoides con una velocidad moderada, con VCL $=51,84 \pm 6,94$ $\mu \mathrm{m} / \mathrm{s}, \mathrm{VSL}=29,46 \pm 6,64 \mu \mathrm{m} / \mathrm{s}$ y VAP $=56,73 \pm 9,66 \mu \mathrm{m} / \mathrm{s}$, pero de trayectoria progresiva $(\mathrm{LIN}=56,73 \pm 9,66 \%$; STR $=80,92 \pm 6,75 \% ; \mathrm{ALH}=2,13 \pm 0,28 \mu \mathrm{m} ; \mathrm{y} \mathrm{BCF}=7,49 \pm 0,56 \mathrm{~Hz}, \mathrm{MP}=62,98 \pm 9,96 \%)$. Del total de los espermatozoides, el 46,83\% fueron asignados a esta subpoblación. La subpoblación 2 estuvo representada por espermatozoides con un movimiento activo, pero no progresivo, reflejado por valores altos de $\mathrm{VCL}=55,67 \pm 9,91 \mu \mathrm{m} / \mathrm{s}$ y $\mathrm{ALH}=2,32 \pm 0,33$ $\mu \mathrm{m}$, y bajos de LIN $(46,95 \pm 12,38 \%)$, STR $(74,78 \pm 9,96 \%)$ y MP $(35,91 \pm 10,96 \%)$. Además, en esta subpoblación se encontró el mayor porcentaje de espermatozoides estáticos $(49,22 \pm 11,56 \%)$. El 14,78\% del total de espermatozoides formó parte de este grupo (Cuadro 10). La subpoblación 3 presentó la menor cantidad de células con un 8,45\%; 
Cuadro 9. Vectores propios de componentes principales (CPs), de parámetros de cinética y movilidad de espermatozoides de verraco empleados en el estudio de la influencia de la composición racial sobre variables reproductivas fenotípicas de calidad espermática, cinética celular y movilidad del semen. Región Huetar Norte, Costa Rica. 2016.

Table 9. Eigenvectors of principal components (PCs) for boar sperm kinetics and motility parameters added in the breed composition study on phenotypic reproductive variables of semen quality, cells kinetics and sperm motility. North Huetar Region, Costa Rica. 2016.

\begin{tabular}{|c|c|c|c|c|}
\hline & \multicolumn{4}{|c|}{ Vectores propios*/a } \\
\hline & 1 & 2 & 3 & 4 \\
\hline LIN & 0,993 & & & \\
\hline WOB & 0,918 & & & \\
\hline STR & 0,860 & & & \\
\hline VSL & 0,712 & 0,666 & & \\
\hline VCL & & 0,913 & & \\
\hline VAP & & 0,883 & & \\
\hline $\mathrm{BCF}$ & & 0,769 & & \\
\hline Estáticos & & & $-0,971$ & \\
\hline Móviles progresivos & & & 0,819 & $-0,492$ \\
\hline Rápidos & & & 0,784 & $-0,491$ \\
\hline Móviles no progresivos & & & & 0,934 \\
\hline Lentos & & & & 0,888 \\
\hline Varianza explicada (\%) & 27,72 & 22,38 & 19,21 & 18,15 \\
\hline
\end{tabular}

* Se expresan las variables más importantes en cada CP. Sólo se presentan vectores propios $>0,4 .{ }^{a}$ Matriz de componente rotado, método de rotación: Varimax con normalización Kaiser. La rotación ha convergido en 5 iteraciones. VCL: velocidad curvilínea; VSL: velocidad rectilínea; VAP: velocidad media; LIN: índice de linearidad; STR: índice de rectitud; WOB: índice de oscilación; ALH: desplazamiento lateral de la cabeza; BCF: frecuencia de entrecruzamiento. MP: motilidad progresiva ( $>5 \mu \mathrm{m} / \mathrm{s})$, MNP: motilidad no progresiva $(<5 \mu \mathrm{m} / \mathrm{s})$, MPR: motilidad progresiva rápida $(\geq 25 \mu \mathrm{m} / \mathrm{s})$. Rápidos $(>45 \mu \mathrm{m} / \mathrm{s})$, Lentos $(10<\mathrm{y}<25 \mu \mathrm{m} / \mathrm{s}) / *$ Expresses the more important variables in each PC. Only eigenvectors $>0.4$ are presented. ${ }^{a}$ Rotated component matrix, rotation method: Varimax with Kaiser normalization. The rotation has converged in 5 iterations. VCL: curvilinear velocity; VSL: straight line velocity; VAP: average path velocity; LIN: linearity of forward progression; STR: straightness; WOB: wobble; ALH: amplitude of lateral head displacement; BCF: beat-cross frequency. MP: progressive motility $(>5 \mu \mathrm{m} / \mathrm{s})$, MNP: non-progressive motility $(<5 \mu \mathrm{m} / \mathrm{s})$, MPR: rapid progressive motility $(\geq 25 \mu \mathrm{m} / \mathrm{s})$. Rapid $(>45 \mu \mathrm{m} / \mathrm{s})$, Slow $(10<\mathrm{y}<25 \mu \mathrm{m} / \mathrm{s})$.

las cuales mostraron una velocidad baja, caracterizada principalmente por mostrar VAP $=37,78 \pm 7,88 \mu \mathrm{m} / \mathrm{s}$, VCL $=57,94 \pm 11,27 \mu \mathrm{m} / \mathrm{s}, \mathrm{VSL}=29,59 \pm 7,56 \mu \mathrm{m} / \mathrm{s}$ y trayectoria progresiva $(\mathrm{LIN}=51,38 \pm 10,37 \% ; \mathrm{STR}=77,89 \pm 7,60 \%$; $\mathrm{WOB}=65,50 \pm 8,02 \% ; \mathrm{MP}=63,40 \pm 10,34 \% ; \mathrm{MPR}=56,91 \pm 16,69 \%)$. La subpoblación 4 estuvo representada por el 29,94\% de la población total, incluyó aquellos espermatozoides con movimiento más rápido pero no progresivo, indicado por los valores más elevados de $\operatorname{VCL}(68,41 \pm 8,71 \mu \mathrm{m} / \mathrm{s})$, $\operatorname{VSL}(29,22 \pm 7,52 \mu \mathrm{m} / \mathrm{s}), \operatorname{VAP}(40,65 \pm 6,83 \mu \mathrm{m} / \mathrm{s})$ y BCF $(8,29 \pm 0,94 \mathrm{~Hz})$, junto con una baja LIN $(42,61 \pm 8,87 \%)$, STR $(70,82 \pm 10,63 \%)$, WOB $(59,51 \pm 6,74 \%)$, MP $(27,73 \pm 11,44)$, MNP $(49,13 \pm 11,11)$ y moderada ALH $(2,70 \pm 0,32 \mu \mathrm{m})$ (Cuadro 10). 
Cuadro 10. Valores medios ( \pm EE) de parámetros de cinética y de movilidad de las diferentes subpoblaciones (SPs*) de espermatozoides de verraco empleados en el estudio de la influencia de la composición racial sobre variables reproductivas fenotípicas de calidad espermática, cinética celular y movilidad del semen. Región Huetar Norte, Costa Rica. 2016.

Table 10. Mean values $( \pm \mathrm{SE})$ of each kinetic and motility parameter corresponding to different subpopulations (SPs*) for boar sperm used in the breed composition study on phenotypic reproductive variables of semen quality, cells kinetics and sperm motility. North Huetar Region of Costa Rica. 2016.

\begin{tabular}{|c|c|c|c|c|}
\hline Variable & SP1 & SP2 & SP3 & SP4 \\
\hline $\mathrm{n} / \%$ & $4880 / 46,83$ & $1540 / 14,78$ & $880 / 8,45$ & $3120 / 29,94$ \\
\hline $\mathrm{VCL}(\mu \mathrm{m} / \mathrm{s})$ & $51,84 \pm 0,54$ & $55,67 \pm 0,96^{\mathrm{b}}$ & $57,94 \pm 1,27^{\mathrm{b}}$ & $68,41 \pm 0,67^{a}$ \\
\hline $\operatorname{VSL}(\mu \mathrm{m} / \mathrm{s})$ & $29,46 \pm 0,46^{\mathrm{a}}$ & $25,91 \pm 0,82^{\mathrm{b}}$ & $29,59 \pm 1,08^{\mathrm{a}}$ & $29,22 \pm 0,57^{\mathrm{a}}$ \\
\hline $\operatorname{VAP}(\mu \mathrm{m} / \mathrm{s})$ & $36,15 \pm 0,44^{\mathrm{a}}$ & $34,25 \pm 0,78^{\mathrm{b}}$ & $37,78 \pm 1,03^{\mathrm{c}}$ & $40,65 \pm 0,55^{\mathrm{c}}$ \\
\hline $\operatorname{LIN}(\%)$ & $56,73 \pm 0,64^{a}$ & $46,96 \pm 1,13^{b}$ & $51,38 \pm 1,50^{\mathrm{b}}$ & $42,61 \pm 0,80^{c}$ \\
\hline STR (\%) & $80,92 \pm 0,55^{\mathrm{a}}$ & $74,78 \pm 0,98^{\mathrm{b}}$ & $77,89 \pm 1,30^{\mathrm{ab}}$ & $70,82 \pm 0,69^{c}$ \\
\hline WOB (\%) & $69,79 \pm 0,50^{\mathrm{a}}$ & $62,00 \pm 0,88^{\mathrm{bc}}$ & $65,50 \pm 1,17^{\mathrm{b}}$ & $59,51 \pm 0,62^{c}$ \\
\hline $\mathrm{ALH}(\mu \mathrm{m})$ & $2,13 \pm 0,07^{\mathrm{c}}$ & $2,32 \pm 0,13^{\mathrm{bc}}$ & $2,98 \pm 0,17^{\mathrm{a}}$ & $2,70 \pm 0,09^{\mathrm{ab}}$ \\
\hline $\mathrm{BCF}(\mathrm{Hz})$ & $7,49 \pm 0,05^{\mathrm{c}}$ & $7,50 \pm 0,09^{\mathrm{bc}}$ & $7,83 \pm 0,12^{b}$ & $8,29 \pm 0,06^{\mathrm{a}}$ \\
\hline $\operatorname{MP}(\%)$ & $62,98 \pm 9,96^{a}$ & $35,91 \pm 10,96^{\mathrm{b}}$ & $63,40 \pm 10,34^{a}$ & $27,73 \pm 11,44^{\mathrm{c}}$ \\
\hline $\operatorname{MNP}(\%)$ & $16,21 \pm 5,21^{\mathrm{bc}}$ & $14,87 \pm 4,94^{\mathrm{c}}$ & $17,08 \pm 5,50^{\mathrm{b}}$ & $49,13 \pm 11,11^{\mathrm{a}}$ \\
\hline MPR (\%) & $39,80 \pm 15,96^{\mathrm{b}}$ & $38,50 \pm 16,91^{\mathrm{b}}$ & $56,91 \pm 16,69^{\mathrm{a}}$ & $34,12 \pm 22,46^{\mathrm{b}}$ \\
\hline Rápidos (\%) & $48,85 \pm 15,18^{b}$ & $27,12 \pm 10,17^{\mathrm{c}}$ & $54,04 \pm 14,19^{a}$ & $15,19 \pm 11,47^{\mathrm{d}}$ \\
\hline Lentos (\%) & $7,85 \pm 4,51^{\mathrm{b}}$ & $6,76 \pm 3,35^{\mathrm{b}}$ & $6,55 \pm 3,94^{b}$ & $25,91 \pm 10,66^{\mathrm{a}}$ \\
\hline Estáticos (\%) & $20,85 \pm 7,86^{\mathrm{b}}$ & $49,22 \pm 11,56^{\mathrm{a}}$ & $19,53 \pm 7,88^{\mathrm{b}}$ & $23,15 \pm 10,27^{\mathrm{b}}$ \\
\hline
\end{tabular}

* Subpoblaciones. VCL: velocidad curvilínea; VSL: velocidad rectilínea; VAP: velocidad media; LIN: índice de linealidad; STR: índice de rectitud; WOB: índice de oscilación; ALH: desplazamiento lateral de la cabeza; BCF: frecuencia de entrecruzamiento. EE: error estándar. MP: motilidad progresiva $(>5 \mu \mathrm{m} / \mathrm{s})$, MNP: motilidad no progresiva $(<5 \mu \mathrm{m} / \mathrm{s})$, MPR: motilidad progresiva rápida $(\geq 25$ $\mu \mathrm{m} / \mathrm{s})$. Rápidos $(>45 \mu \mathrm{m} / \mathrm{s})$, Lentos $(10<\mathrm{y}<25 \mu \mathrm{m} / \mathrm{s})$. a,b,c Dentro de fila, valores con diferente superíndice presentan diferencias significativas $\mathrm{P}<0,05 / *$ Subpopulation. VCL: curvilinear velocity; VSL: straight line velocity; VAP: average path velocity; LIN: linearity of forward progression; STR: straightness; WOB: wobble; ALH: amplitude of lateral head displacement; BCF: beat-cross frequency. LSM: Least squares mean; SE: standard error. MP: progressive motility $(>5 \mu \mathrm{m} / \mathrm{s})$, MNP: non-progressive motility $(<5$ $\mu \mathrm{m} / \mathrm{s})$, MPR: rapid progressive motility $(\geq 25 \mu \mathrm{m} / \mathrm{s})$. Rapid $(>45 \mu \mathrm{m} / \mathrm{s})$, Slow $(10<\mathrm{y}<25 \mu \mathrm{m} / \mathrm{s})$. a,b,c Within the same row, values with different superscripts show significant difference at $\mathrm{P}<0.05$.

\section{Discusión}

El análisis de semen asistido por computadora es útil y necesario en los centros de inseminación artificial y en las empresas dedicadas a la venta de dosis seminales, porque optimiza la producción de las mismas (Broekhuijse et al., 2012) y garantiza una objetiva evaluación de la calidad seminal. En estudios anteriores se ha descrito que la calidad seminal es multifactorial. La especie, raza, edad, época, nutrición, salud, estímulo sexual, estrés y tamaño testicular son factores que pueden influir en la calidad del semen (Ciereszko et al., 2000; Kawęcka et al., 2008; Kondracki et al., 2012). En relación con el efecto del verraco y el grupo racial en la evaluación de la calidad del semen, los resultados en este trabajo mostraron diferencias $(\mathrm{P}<0,05)$ entre razas y líneas genéticas estudiadas. Los verracos estudiados presentaron más variabilidad en los caracteres relacionados con la cantidad de semen (volumen, concentración y número total de espermatozoides) que en rasgos de calidad del mismo (motilidad espermática). 
Las razas difirieron en el volumen de sus eyaculados $(\mathrm{P}<0,05)$, esto influyó en el número total de espermatozoides, lo cual es consistente con los resultados de Wolf y Smital (2009) y Tăpăloagă et al. (2013), quienes encontraron que había diferencias numéricas, pero no estadísticamente significativas. El volumen de eyaculado difirió entre verracos de diferentes razas y composición genética, la raza Yorkshire presentó el mayor volumen y la Duroc el menor $(\mathrm{P}<0,05)$.

El volumen eyaculado promedio en las razas Landrace, Yorkshire y la línea genética A, obtenido fue consistente con los resultados encontrados por Wolf y Smital (2009) y Tăpăloagă et al. (2013), pero mayores que los volúmenes de eyaculado encontrados por Savić et al. (2013). El volumen promedio de eyaculado en el presente estudio fue aproximadamente $231,13 \pm 97,40 \mathrm{ml}$ para verracos de edades medias de 24,41 $\pm 10,90$ meses. No hubo evidencia suficiente para afirmar que alguna raza fue superior en todos los rasgos observados. Un mayor volumen de eyaculado en verracos Large White fue encontrado por Knecht et al. (2014), sin embargo, cuando se compararon con otras razas, estos verracos presentaban valores bajos y poco deseables para otras variables espermáticas como concentración. Esto plantea la idea de que los eyaculados con volúmenes mayores, pueden actuar de forma contraproducente en la calidad espermática al presentar mayores porcentajes de espermatozoides no funcionales (Martinez-Alborcia et al., 2012) o con menor movilidad. En este trabajo, los verracos de la raza Yorkshire presentaron el mayor volumen de eyaculado, pero presentaron menor porcentaje de células con movimiento rápido y porcentajes altos de espermatozoides con movimiento medio y lento, sin embargo, esto debe ser estudiado más detalladamente. El valor promedio de espermatozoides totales en el eyaculado para las razas evaluadas fue de $77,85 \times 10^{9}$ espermatozoides. Sin embargo, al observar los valores del número total de espermatozoides, hubo verracos superiores a la media (Y, L, PD y LA) y otros presentaron valores inferiores (LB y D). En el presente trabajo, los verracos de la raza Duroc estuvieron muy por debajo del promedio, lo que coincide con lo reportado en estudios previos (Burian et al., 1987; Smital, 2009). El aumento de la actividad espermatogénica se correlaciona positivamente con el número total de espermatozoides en el eyaculado, y esto influye en el número de dosis seminales (Wysokińska et al., 2009; Adamiak et al., 2010; Knecht et al., 2013). Los autores coinciden en que, ninguna raza sobresale en todas las características del semen (Kennedy y Wilkins, 1984; Oh et al., 2006; Smital et al., 2004).

La concentración de espermatozoides en el eyaculado varió entre los verracos de distintas razas y líneas genéticas, aunque las diferencias no fueron estadísticamente significativas, coincidiendo con Wolf y Smital (2009) y Tăpăloagă et al. (2013), quienes no reportaron diferencias significativas en las concentraciones espermáticas de los verracos de diferentes razas. Se ha descrito que los verracos que tenían mayor concentración de espermatozoides presentaban menor volumen de eyaculado, mientras otros reproductores tenían el mayor volumen y la menor concentración de espermatozoides (Tăpăloagă et al., 2013). En el presente trabajo se registraron resultados similares, los verracos de la raza Duroc tuvieron el menor volumen de eyaculado $(\mathrm{P}<0,05)$, pero con una alta concentración. Estos hallazgos sugieren que el menor volumen de eyaculación puede ser compensado por una mayor concentración de espermatozoides (Knecht et al., 2014; Žaja et al., 2016). La concentración de espermatozoides de diferentes razas de verracos y líneas genéticas obtenida, varió entre 0,325 y $0,357 \times 10^{9} / \mathrm{ml}$ $\left(325-357 \times 10^{6} / \mathrm{ml}\right)$ y fueron consistentes con los datos obtenidos por Kunavongkrit et al. (2005), Tăpăloagă et al. (2013) y Knecht et al. (2014).

Los valores bajos en el total de espermatozoides por eyaculado en la raza Duroc coinciden con los reportados por Kawęcka et al. (2008) en verracos de la misma raza a los 270 días de edad; sin embargo, difirieron de los reportados por Smital (2009). El número total de espermatozoides no fue estadísticamente diferente en los verracos Landrace, $\mathrm{F}_{1}$ Pietrain*Duroc, así como las líneas A y B, mientras que el mayor número de espermatozoide totales lo presentó la raza Yorkshire $\left(110,5 \times 10^{9}\right)$. Resultados similares en el cruce $\mathrm{F}_{1}$ Pietrain*Duroc fueron obtenidos por Ciereszko et al. (2000) y Knecht et al. (2014) y muy superiores con respecto a los reportados por Kawęcka et al. (2008), con valores entre 20,6 y 28,4 x $10^{9}$ espermatozoides. En el presente estudio, los valores del número 
total de espermatozoides por eyaculado fueron menores que los de Smital (2009) y mayores que los de Kawęcka et al. (2008) en razas y manejos reproductivos similares, por lo que, es necesario más investigación de las posibles fuentes de variación en esta característica a nivel de raza y edad de los verracos.

Las diferencias entre razas en la calidad seminal se han demostrado anteriormente (Conlon y Kennedy, 1978; Kennedy y Wilkins, 1984; Ciereszko et al., 2000; Sonderman y Luebbe, 2008; Smital, 2009). La VSL fue mayor en las razas Duroc, Yorkshire y la línea A. Este es un parámetro importante en los centros de inseminación artificial para el procesado de las dosis seminales. En los trabajos de Holt et al. (1997) y Broekhuijse et al. (2012), este parámetro mostró una relación positiva con la fertilidad del verraco. Una mayor velocidad rectilínea podría permitir a los espermatozoides fertilizar mejor el ovocito (Liu et al., 1991). El índice de linealidad (LIN) presentó una diferencia relevante entre los grupos raciales LB y PD (7,47\%), mientras que, para el índice de rectitud (STR), las razas PD y L presentaron los porcentajes más bajos. La VSL y los índices LIN y STR, se han asociado con la capacidad fertilizante de los espermatozoides y se ha sugerido que valores elevados de VSL, LIN y STR, podrían ser relevantes en el momento del transporte a través del tracto genital femenino y, finalmente, la penetración de la matriz del cumulus (Martínez-Rodríguez et al., 2012).

No se encontraron diferencias $(\mathrm{P}>0,05)$ en la ALH de las diferentes razas estudiadas, sin embargo, la línea B, presentó el valor menor. En el semen destinado para la inseminación artificial este valor debe ser bajo según Leahy y Gadella (2011). En otros trabajos, la ALH se ha descrito como un parámetro de motilidad importante durante la capacitación espermática, y requerido para lograr la penetración de las barreras de fertilización que rodean al ovocito, como las capas de células del cumulus oophorus y la zona pelúcida (Gadea, 2005).

La BCF es una característica del espermatozoide que permite estimar cambios significativos en el patrón de movilidad del flagelo (Sellés et al., 2003), pero la estimación está asociada con el número de imágenes por segundo que se pueden obtener con un sistema CASA. La velocidad de captura de imágenes del sistema CASA en este trabajo fue de $25 \mathrm{~Hz}$ pudiendo llegar hasta $60 \mathrm{~Hz}$ (especificaciones del sistema ISASv1). Los resultados del presente estudio demostraron una clara diferenciación $(\mathrm{P}<0,05)$ entre las líneas genéticas A y $\mathrm{B}$, donde la primera presentó el valor menor. De acuerdo con Broekhuijse et al. (2012), en el momento de la evaluación de la motilidad (semen fresco), se prefiere que las células espermáticas aún no tengan frecuencias de batido elevadas, y permanecer así hasta que se produzca la penetración de la zona pelúcida (Gil et al., 2009).

La raza Landrace presentó el menor porcentaje de células con movimiento rápido y mayores porcentajes de células con movimiento medio y lento; además, fue la raza que presentó mayor cantidad de espermatozoides estáticos. En contraposición, la raza Duroc, PD y LB, presentaron mayores porcentajes $(\mathrm{P}<0,05)$ de células con movimiento rápido, sin detectarse diferencias significativas entre ellas. En términos generales, se podría considerar que los tratamientos que presenten los mayores resultados de velocidad son los mejores (Amann y Katz, 2004), sin embargo, no existe evidencia biológica para apoyar esta suposición. Las diferencias individuales entre verracos y entre grupos raciales son independientes del método de evaluación del semen (Broekhuijse et al., 2012), y las diferencias en la motilidad y otras características seminales son función de los grupos raciales (Sonderman y Luebbe, 2008), por lo que, no puede afirmarse categóricamente que las razas y/o líneas de verracos con menor movilidad espermática tengan automáticamente una fertilidad menor.

Las diferencias más relevantes $(\mathrm{P}<0,05)$ en la MTOT y MP se presentaron entre la raza Landrace y el $\mathrm{F}_{1}$ Pietrain*Duroc, donde el cruce $\mathrm{F}_{1}$ presentó los valores mayores. Valores altos de MTOT y MP, se asociaron a una mayor proporción de espermatozoides con un mayor potencial de membrana mitocondrial que, influye en el estado energético de las mitocondrias de los espermatozoides y se relaciona con las características de la motilidad (Fraser et al., 2007; Dziekońska et al., 2009; Davila et al., 2016; Wasilewska y Fraser, 2017). Resultados similares a los del presente estudio, para la motilidad total de los espermatozoides, fueron obtenidos por Gerfen et al. (1994) y Wolf y Smital (2009). La motilidad espermática (Tardif et al., 1999) y la cinética (Holt et al., 1997) se relacionan con la fertilidad, aunque en otros trabajos se han reportado resultados inconsistentes (Oh et al., 2010). 
Un mayor porcentaje de MTOT fue encontrado en verracos "terminales" con respecto a los "abuelos" $(75,74 \pm 0,62$ vs $71,36 \pm 1,63 \%$, respectivamente). Sin embargo, no hubo diferencias $(\mathrm{P}>0,05)$ en el porcentaje de células con movimiento progresivo (MP), ni en el porcentaje de espermatozoides con movimiento progresivo categorizado como rápido (MPR).

Algunos autores consideran el efecto de la influencia de la raza en la calidad del esperma en los criterios de selección utilizados para la mejora genética del porcino (Flowers, 1997). Los sistemas actuales de índices de selección en las líneas maternas (Y, L) toman en cuenta los rasgos reproductivos, mientras que, en las líneas paternas (LA, LB, D, ) utilizadas para producción de engorde de cerdos, la selección se centra en rasgos terminales con un potencial impacto negativo en caracteres maternos. Esto podría tener un impacto negativo en la calidad del esperma del verraco, ya que probablemente un componente genético podría ser responsable de las diferencias en las características de los espermatozoides (Hoflack et al., 2007). Debido a que, en la industria de la inseminación artificial la capacidad de los verracos seleccionados de producir eyaculados de alta calidad y cantidad es fundamental desde el punto de vista económico, la selección de los verracos debe ponderarse por los caracteres maternos y terminales para lograr resultados óptimos (Knox, 2014).

No se encontró una correlación lineal significativa entre la movilidad o concentración del semen y la edad de los verracos. El volumen de eyaculado y el número total de espermatozoides fue mayor en los verracos adultos. En otros trabajos se ha reportado una relación significativa entre la edad de los verracos jóvenes y la movilidad del semen diluido (Schulze et al., 2014). En un estudio con verracos Yorkshire y Landrace con edades entre 6,5 y 10 meses de edad, se reportó aumento de la motilidad espermática durante el primer año de la vida del verraco (Swierstra, 1974). En el presente trabajo, los verracos menores de ocho meses mostraron una tendencia con valores más elevados de motilidad total, aunque no significativos.

La VSL y la VAP fueron menores ( $\mathrm{P}<0,05)$ en los verracos jóvenes ( $<8$ meses), sin embargo, la VCL fue mayor $(\mathrm{P}<0,05)$ en los de edad intermedia $(\geq 8 \mathrm{y}<18$ meses), y no se detectaron diferencias entre los verracos menores de 8 meses y los mayores de 18 meses. Estos resultados plantean la idea de que es necesario considerar la diferenciación por edad para verracos muy jóvenes (menores de ocho meses) con respecto a los parámetros cualitativos de la motilidad de los espermatozoides (Schulze et al., 2014). Los verracos jóvenes, en comparación con los adultos, frecuentemente presentan una calidad de esperma más variable, lo que coincide con lo encontrado en este trabajo, se considera preferible proceder con la selección solo después de tres o cuatro evaluaciones seminales, ya que, la pubertad de un verraco comienza a la edad de 4,5-5,5 meses, mientras que, su madurez sexual a los 7-8 meses (Schinckel et al., 1983).

En el análisis de componentes principales se identificaron cuatro componentes que en total explicaron 87,47\% de la variación total observada. Existe evidencia de que mayores VAP, VSL y BCF se asocian positivamente con la integridad de la membrana del flagelo (Grieblová et al., 2017), lo que coincide con lo encontrado en el presente estudio en el segundo componente principal. Este hallazgo sugiere que, la integridad de la membrana del flagelo podría promover un movimiento lineal rápido, que puede facilitar la progresión del espermatozoide a través del tracto reproductivo femenino (Grieblová et al., 2017). En el presente estudio se determinaron cuatro subpoblaciones espermáticas en semen fresco. La primera subpoblación agrupó a los espermatozoides con movimiento moderado pero muy progresivo, la segunda incluyó células con movimiento activo, pero no progresivo, la tercera se asoció con células con movimiento reducido pero progresivo y la cuarta subpoblación incluyó células con movimiento activo, pero no progresivo. Estos datos fueron similares a los obtenidos por Abaigar et al. (1999), Cremades et al. (2005), Flores et al. (2008, 2009) y Estrada et al. (2017), con semen refrigerado y capacitado, lo que sugiere que, la presencia de tres a cuatro subpoblaciones según los parámetros cinéticos, es una característica inherente de los espermatozoides del verraco.

Tan solo una fracción mínima con respecto al total producido de espermatozoides, es necesaria para fertilizar los ovocitos de una cerda (Holt y van-Look, 2004), y todavía no se conoce del todo este riguroso proceso 
selectivo. Sin embargo, aunque la contractilidad uterina desempeña un papel importante para el transporte de espermatozoides dentro de la cerda, no se debe obviar el papel de la motilidad per se del semen (Langendijk et al., 2005; Yeste y Castillo-Marín, 2013). Es reciente el estudio de las subpoblaciones de células espermáticas dentro de un eyaculado, y no se ha establecido una relación clara entre subpoblaciones móviles y el rendimiento reproductivo en semen fresco (Quintero-Moreno et al., 2004), o si los patrones de motilidad o anomalías específicas influyen en la fertilidad del verraco (Didion, 2008). Además, Braundmeier y Miller (2001) sugirieron que los espermatozoides que fertilizan los ovocitos in vivo pueden ser una subpoblación reducida y altamente seleccionada y eficiente, pero no representativa de la media de células analizadas en el eyaculado con los sistemas CASA. Debe continuarse con el estudio de subpoblaciones y asociarlo con características de fertilidad para entender los procesos de selectividad espermática.

\section{Conclusiones}

El grupo racial influyó en el volumen de eyaculado y en el número total de espermatozoides, lo que repercutió en el número de dosis seminales producidas. No se identificó una relación directa y positiva entre la velocidad y la progresividad y/o linealidad de los diferentes grupos raciales. La motilidad total fue útil como primera aproximación de la calidad seminal, pero debe acompañarse de otros parámetros indicadores como la motilidad progresiva y la velocidad de los espermatozoides clasificados como progresivos, en donde hay claras diferencias en la composición racial. Las clasificaciones del movimiento espermático permiten diferenciar los grupos raciales en la calidad de un eyaculado y asociarlo con la naturaleza del reproductor y la selección de caracteres reproductivos dentro de la raza. La edad del verraco fue un factor importante en la evaluación de la calidad seminal, por lo que, verracos muy jóvenes presentaron alta variabilidad en los parámetros seminales con respecto a verracos en edades intermedias y adultos. Las subpoblaciones espermáticas descartan la idea general del eyaculado como un "todo" y permiten determinar conjuntos celulares afines por patrones de movimiento y progresividad.

\section{Agradecimientos}

Los autores agradecen a la Fundación para el Fomento y la Promoción de la Investigación y Transferencia de Tecnología Agropecuaria y el PITTA cerdos del Ministerio de Agricultura y Ganadería de Costa Rica, y a la Vicerrectoría de Investigación y Extensión del Instituto Tecnológico de Costa Rica, por el apoyo recibido. Se agradece también a los productores de la Región Huetar Norte por facilitar sus unidades de producción y a los estudiantes de la Escuela de Agronomía del Instituto Tecnológico de Costa Rica, J. Solís, A. Vega, L. Víquez, K. Arguedas, F. Álvarez y J.P. Gamboa, por su desempeño como asistentes del proyecto de investigación.

\section{Literatura citada}

Abaigar, T., W.V. Holt, R.A.P. Harrison, and G. del-Barrio. 1999. Sperm subpopulations in boar (Sus scrofa) and gazelle (Gazella dama mhorr) semen as revealed by pattern analysis of computer-assisted motility assessments. Biol. Reprod. 60:32-41. doi:10.1095/biolreprod60.1.32

Adamiak, A., S. Kondracki, and A. Wysokińska. 2010. Influence of season of the year on physical properties of ejaculates from Polish Large White and Polish Landrace boars. Roczniki Naukowe Zootech. 37:159-167. 
Amann, R.P., and D.F. Katz. 2004. Reflections on CASA after 25 years. J. Androl. 25:317-324. doi:10.1002/j.1939-4640.2004. tb02793.X

Bompart, D., A. García-Molina, A. Valverde, C. Caldeira, J. Yániz, M. Núñez de Murga, and C. Soler. 2018. CASA-Mot technology: how results are affected by the frame rate and counting chamber. Reprod Fertil Dev. doi:10.1071/RD17551

Braundmeier, A.G., and D.J. Miller. 2001. The search is on: finding accurate molecular markers of male fertility. J. Dairy Sci. 84:1915-1925. doi:10.3168/jds.S0022-0302(01)74633-4

Broekhuijse, M.L., E. Šoštarić, H. Feitsma, and B.M. Gadella. 2012. Application of computer-assisted semen analysis to explain variations in pig fertility. J. Anim. Sci. 90:779-789. doi:10.2527/jas.2011-4311

Burian, F., S. Buchta, V. Řehák, M. Sládek, a I. Schmidt. 1987. Analýza spermatologických ukazatelů kanců plemen bílé ušlechtilé, landrase a duroc a hybridnı́ch kanců SL 98 F1, F2 a F3 generace. Živoč. Výr. 32:1097-1103.

Ciereszko, A., J.S. Ottobre, and J. Glogowski. 2000. Effects of season andbreed on sperm acrosin activity and semen quality of boars. Anim. Reprod. Sci. 64:89-96. doi:10.1016/S0378-4320(00)00194-9

Conlon, P.D., and B.W. Kennedy. 1978. A comparison of crossbred and purebred boars for semen and reproductive characteristics. Can. J. Anim. Sci. 58:63-70. doi:10.4141/xjas78-009

Cremades, T., J. Roca, H. Rodriguez-Martinez, T. Abaigar, J.M. Vazquez, and E.A. Martinez. 2005. Kinematic changes during the cryopreservation of boar spermatozoa. J. Androl. 26:610-618. doi:10.2164/jandrol.05028

Davila, M.P., P.M. Muñoz, J.M. Bolaños, T.A. Stout, B.M. Gadella, J.A. Tapia, C.B. da-Silvia, C.O. Ferrusola, and F.J. Peña. 2016. Mitochondrial ATP is required for the maintenance of membrane integrity in stallion spermatozoa, whereas motility requires both glycolysis and oxidative phosphorylation. Reproduction 152:683-694. doi:10.1530/REP-16-0409

Didion, B.A. 2008. Computer-assisted semen analysis and its utility for profiling boar semen samples. Theriogenology 70:13741376. doi:10.1016/j.theriogenology.2008.07.014

Dyck, M.K., G.R. Foxcroft, S. Novak, A. Ruiz-Sanchez, J. Patterson, and W.T. Dixon. 2011. Biological markers of boar fertility. Reprod. Domest. Anim. 46:55-58. doi:10.1111/j.1439-0531.2011.01837.x

Dziekońska, A., L. Fraser, and J. Strzeżek. 2009. Effect of different storage temperatures on the metabolic activity of spermatozoa following liquid storage of boar semen. J. Anim. Feed. Sci. 18:638-649. doi:100.22358/jafs/66438/2009

Estrada, E., M.M. Rivera-del-Álamo, J.E. Rodríguez-Gil, and M. Yeste. 2017. The addition of reduced glutathione to cryopreservation media induces changes in the structure of motile subpopulations of frozen-thawed boar sperm. Cryobiology 78:56-64. doi:10.1016/j.cryobiol.2017.07.002

Flores, E., J.M. Fernández-Novell, A. Peña, and J.E. Rodríguez-Gil. 2009. The degree of resistance to freezing-thawing is related to specific changes in the structures of motile sperm subpopulations and mitochondrial activity in boar spermatozoa. Theriogenology 72:784-797. doi:10.1016/j.theriogenology.2009.05.013

Flores, E., E. Taberner, M.M. Rivera, A. Peña, T. Rigau, J. Miró, and J.E. Rodríguez-Gil. 2008. Effects of freezing/ thawing on motile sperm subpopulations of boar and donkey ejaculates. Theriogenology 70:936-945. doi:10.1016/j. theriogenology.2008.05.056

Flowers, W.L. 1997. Management of boars for efficient semen production. J. Reprod. Fertil. Suppl. 52:67-78.

Fraser, L., A. Dziekońska, R. Strzezek, and J. Strzezek. 2007. Dialysis of boar semen prior to freezing thawing: its effects on post-thaw sperm characteristics. Theriogenology 67:994-1003. doi:10.1016/j.theriogenology.2006.12.002

Gadea, J. 2005. Sperm factors related to in vitro and in vivo porcine fertility. Theriogenology 63:431-444. doi:10.1016/j. theriogenology.2004.09.023 
Gerfen, R.W., B.R. White, M.A. Cotta, and M.B. Wheeler. 1994. Comparison of the semen characteristics of fengjing, meishan and Yorkshire boars. Theriogenology 41:461-469. doi:10.1016/0093-691X(94)90082-T

Gil, M.C., M. García-Herreros, F.J. Barón, I.M. Aparicio, A.J. Santos, and L.J. García-Marín. 2009. Morphometry of porcine spermatozoa and its functional significance in relation with the motility parameters in fresh semen. Theriogenology 71:254-263. doi:10.1016/j.theriogenology.2008.07.007

Grieblová, A., E. Pintus, and J. Ros-Santaella. 2017. Integrity of head and tail plasmalemma is associated with different kinetic variables in boar sperm. Anim. Reprod. Sci. 184:218-227. doi:10.1016/j.anireprosci.2017.07.020

Hoflack, G., G. Opsomer, T. Rijsselaere, A. Van-Soom, D. Maes, A. de-Kruif, and L. Duchateau. 2007. Comparison of computer-assisted sperm motility analysis parameters in semen from Belgian blue and Holstein-Friesian bulls. Reprod. Domest. Anim. 42:153-161. doi:10.1111/j.1439-0531.2006.00745.x

Holt, C., W.V. Holt, H.D. Moore, H.C. Reed, and R.M. Curnock. 1997. Objectively measured boar sperm motility parameters correlate with the outcomes of on-farm inseminations: results of two fertility trials. J. Androl. 18:312-323. doi:10.1002/j.1939-4640.1997.tb0195.x

Holt, W.V., and J.W. Van-Look. 2004. Concepts in sperm heterogeneity, sperm selection and sperm competition as biological foundations for laboratory test of semen quality. Reproduction 127:527-535. doi:10.1530/rep.1.00134

Kawęcka, M., A. Pietruszka, E. Jacyno, R. Czarnecki, and M. Kamyczek. 2008. Quality of semen of young boars of the breeds Pietrain and Duroc and their reciprocal crosses. Arch. Tierz. 51:42-54. doi:10.5194/aab-51-42-2008

Kennedy, B.W., and J.N. Wilkins. 1984. Boar, breed and environmental factors influencing semen characteristics of boars used in artificial insemination. Can. J. Anim. Sci. 64:833-843. doi:10.4141/cjas84-097

Knecht, D., S. Środoń, and K. Duziński. 2014. The influence of boar breed and season on semen parameters. S. Afr. J. Anim. Sci. 44:1-9. doi:10.4314/sajas.v44i1.1

Knecht, D., S. Środoń, K. Szulc, and K. Duziński. 2013. The effect of photoperiod on selected parameters of boar semen. Liv. Sci. 157:364-71. doi:10.1016/j.livsci.2013.06.027

Knox, R.V. 2014. Impact of swine reproductive technologies on pig and global food production. In: G.C. Lamb, and N. DiLorenzo, editors, Current and future reproductive technologies and world food production. Springer, NY, USA. p. 131-160.

Kondracki, S., M. Iwanina, A. Wysokińska, and M. Huszno. 2012. Comparative analysis of Duroc and Pietrain boar sperm morphology. Acta Vet. Brno 81:195-199. doi:10.2754/avb201281020195

Kunavongkrit, A., A. Suriyasomboom, N. Lundeheim, T.W. Heard, and S. Einarsson. 2005. Management and sperm production of boars under differing environmental conditions. Theriogenology 63:657-667. doi:10.1016/j.theriogenology.2004.09.039

Langendijk, P., N.M. Soede, and B. Kemp. 2005. Uterine activity, sperm transport, and the role of boar stimuli around insemination in sows. Theriogenology 63:500-513. doi:10.1016/j.theriogenology.2004.09.027

Leahy, T., and B.M. Gadella. 2011. Sperm surface changes and physiological consequences induced by sperm handling and storage. Reproduction 142:759-778. doi:10.1530/REP-11-0310

Liu, D.Y., G.N. Clarke, and H.W. Gordon-Baker. 1991. Relationship between sperm motility assessed with the Hamilton-Thorn motility analyzer and fertilization rates in vitro. J. Androl. 12:231-239. doi:10.1002/j.1939-4640.1991.tb00258.x

López-Rodríguez, A., T. Rijsselaere, J. Beek, P. Vyt, A. Van-Soom, and D. Maes. 2013. Boar seminal plasma components and their relation with semen quality. Syst. Biol. Reprod. Med. 59:5-12. doi:10.3109/19396368.2012.725120

Martinez-Alborcia, M.J., A. Valverde, I. Parrilla, J.M. Vazquez, E.A. Martinez, and J. Roca. 2012. Detrimental effects of non-functional spermatozoa on the freezability of functional spermatozoa from boar ejaculate. PLoS ONE 7(5):e36550. doi:10.1371/journal.pone.0036550 
Martínez-Rodríguez, C., M. Alvarez, L. Ordás, C.A. Chamorro, F. Martinez-Pastor, L. Anel, and P. de-Paz. 2012. Evaluation of ram semen quality using polyacrylamide gel instead of cervical mucus in the sperm penetration test. Theriogenology 77:1575-86. doi:10.1016/j.theriogenology.2011.11.026

Oh, S.A., Y.J. Park, Y.A. You, E.A. Mohamed, and M.G. Pang. 2010. Capacitation status of stored boar spermatozoa is related to litter size of sows. Anim. Reprod. Sci. 121:131-138. doi:10.1016/j.anireprosci.2010.05.019

Oh, S.H., M.T. See, T.E. Long, and J.M. Galvin. 2006. Estimates of genetic correlations between production and semen traits in boar. Asian-Aust. J. Anim. Sci. 19:160-164. doi:10.5713/ajas.2006.160

Okere, C., A. Joseph, and M. Ezekwe. 2005. Seasonal and genotype variations in libido, semen production and quality in artificial insemination boars. J. Anim. Vet. Adv. 4:885-888.

Quintero-Moreno, A., T. Rigau, and J.E. Rodríguez-Gil. 2004. Regression analyses and motile sperm subpopulation structure study as improving tools in boar semen quality analysis. Theriogenology 61:673-690. doi:10.1016/S0093691X(03)00248-6

Robinson, J.A.B., and M.M. Buhr. 2005. Impact of genetic selection on management of boar replacement. Theriogenology 63:668-678. doi:10.1016/j.theriogenology.2004.09.040

Savić, R., M. Petrović, D. Radojković, Č. Radović, and N. Parunović. 2013. The effect of breed, boar and season on some properties of sperm. Biotechnol. Anim. Husb. 29:299-310. doi:10.2298/BAH1302299S

Schinckel, A., R.K. Johnson, R.A. Pumfrey, and D.R. Zimmerman. 1983. Testicular growth in boars of different genetic lines and its relationship to reproductive performance. J. Anim. Sci. 56:1065-1076. doi:10.2527/jas1983.5651065x

Schulze, M., S. Buder, K. Rüdiger, M. Beyerbach, and D. Waberski. 2014. Influences on semen traits used for selection of young AI boars. Anim. Reprod. Sci. 148:164-170. doi:10.1016/j.anireprosci.2014.06.008

Sellés, E., J. Gadea, R. Romar, C. Matás, and S. Ruiz. 2003. Analysis of in vitro fertilizing capacity to evaluate the freezing procedures of boar semen and to predict the subsequent fertility. Reprod. Domest. Anim. 38:66-72. doi:10.1046/j.14390531.2003.00406.x

Smital, J. 2009. Effects influencing boar semen. Anim. Reprod. Sci. 110:335-346. doi:10.1016/j.anireprosci.2008.01.024

Smital, J., L.L. De-Sousa, and A. Mohsen. 2004. Differences among breeds and manifestation of heterosis in AI boar sperm output. Anim. Reprod. Sci. 80:121-130. doi:10.1016/S0378-4320(03)00142-8

Sonderman, J.P., and J.J. Luebbe. 2008. Semen production and fertility issues related to differences in genetic lines of boars. Theriogenology 70:1380-1383. doi:10.1016/j.theriogenoly.2008.08.009

Swierstra, E.E. 1974. A comparison of regular ejaculation with sexual rest on semen characteristics and reproductive organ weights in young boars. J. Anim. Sci. 39:575-581.

Tăpăloagă, P.R., A. Șonea, A. Iancu, and E. Mitrănescu. 2013. Researches regarding age, breed and collecting season influence in quality and quantity boars semen. Sci. Pap. Ser. D. Anim. Sci. 56:161-165.

Tardif, S., J.P. Laforest, N. Cormier, and J.L. Bailey. 1999. The importance of porcine sperm parameters on fertility in vivo. Theriogenology 52:447-459. doi:10.1016/S0093-691X(99)00142-9

Tsakmakidis, I.A., A.G. Lymberopoulos, and T.A. Khalifa. 2010. Relationship between sperm quality traits and field-fertility of porcine semen. J. Vet. Sci. 11:151-154. doi:10.4142/jvs.2010.11.2.151

Verstegen, J., M. Iguer-Ouada, and K. Onclin. 2002. Computer assisted semen analyzers in andrology research and veterinary practice. Theriogenology 57:149-179. doi:10.1016/S0093-691X(01)00664-1 
Wasilewska, K., and L. Fraser. 2017. Boar variability in sperm cryo-tolerance after cooling of semen in different long-term extenders at various temperatures. Anim. Reprod. Sci. 185:161-173. doi:10.1016/j.anireprosci.2017.08.016

WHO (World Health Organization). 2010. Laboratory manual for the examination and processing of human semen. 5th ed. Cambridge University Press, Cambridge, GBR.

Wolf, J., and J. Smital. 2009. Effects in genetic evaluation for semen traits in Czech Large White and Czech Landrace boars. Czech J. Anim. Sci. 54:349-358.

Wysokińska, A., S. Kondracki, D. Kowalewski, A. Adamiak, and E. Muczyńska. 2009. Effect of seasonal factors on the ejaculate properties of crossbred Duroc x Pietrain and Pietrain x Duroc boars as well as purebred Duroc and Pietrain boars. Bull. Vet. Inst. Pulawy 53:677-685.

Yeste, M., and M. Castillo-Martín. 2013. Boar spermatozoa within the Uterus. In: S. Bonet et al., editors, Boar reproduction: Fundamentals and new biotechnological trends. Springer, Berlin, GER. p. 101-168.

Žaja, I.Ž., M. Samardžija, S. Vince, I. Majić-Balić, M. Vilić, D. Đuričić, and S. Milinković-Tur. 2016. Influence of boar breeds or hybrid genetic composition on semen quality and seminal plasma biochemical variables. Anim. Reprod. Sci. 164:169176. doi:10.1016/j.anireprosci.2015.11.027 